\title{
Free convection of Walter's fluid flow in a vertical double-passage wavy channel with heat source
}

\author{
J. Prathap Kumar, J.C. Umavathi*, and H. Prema \\ Department of Mathematics, Gulbarga University, Gulbarga, Karnataka, INDIA. \\ "Corresponding Author: jc_uma11@yhaoo.com
}

\begin{abstract}
The steady two-dimensional free convection flow of a Walter's fluid (Model B') in a vertical double passage wavy channel has been investigated analytically in the presence of heat source. The channel is divided into two passages by means of thin, perfectly conductive plane baffle and each stream will have its own pressure gradient and hence the velocity will be individual in streams. The governing equations of the fluid and the heat transfer have been solved subject to the relevant boundary conditions by assuming that the solution consists of two parts; a mean part and disturbance or perturbed part. To obtain the perturbed part of the solution, the long wave approximation has been used and to solve the mean part, well known approximation used by Ostrach has been utilized. Numerical results are presented graphically for the distribution of velocity and temperature fields for varying physical parameters such as baffle position, Grashof number, wall temperature ratio, viscoelastic parameter and product of non-dimensional wave number and space co-ordinate at different positions of the baffle. The relevant flow and heat transfer characteristics namely, skin friction and the rate of heat transfer at both the walls has been discussed in detail.
\end{abstract}

Keywords: vertical wavy channel; baffle; double passage; heat source.

\section{Introduction}

The corrugated wall channel is one of the several devices employed for enhancing the heat transfer efficiency of industrial transport processes. It is necessary to study the heat transfer from irregular surfaces because irregular surfaces are often present in many applications. Mixed convection from wavy surfaces can be used for transferring heat in several heat transfer devices, such as flat-plate solar collectors and flat-plate condensers in refrigerators. The presence of roughness elements disturbs the flow past surfaces and alerts the heat transfer rate. Viscous flow in wavy channels was first treated analytically by Burns and Parks (1962). The solution was obtained by expressing the stream function in a Fourier series under the assumption of Stokes flow. Vajravelu (1980) studied the flow and heat transfer effects accounting for the convection contributions by a perturbation method using the long-wave approximation, where the solution consist of a mean part and perturbed part.

Yao (1983) proposed a simple transformation to study the natural convection heat transfer from isothermal vertical surfaces, such as sinusoidal surfaces, in Newtonian fluids. Chiu and Chou (1994) studied the transient and steady natural convection along a vertical wavy surface in micropolar fluids. They have found that increasing the micropolar parameter results in decreasing the heat transfer rate. Rees and Pop (1994) studied the free convection flow along a vertical wavy surface with constant wall temperature. Rees and Pop (1995) also studied the natural convection flow along a vertical wavy surface with uniform wall flux.

Wang and Vanka (1995) determined the rates of heat transfer for a flow through a periodic array of wavy passage. They observed that for the steady-flow regime, the average Nusselt numbers for the wavy-wall channel were only slightly larger than those for a parallel-plate channel. However, in the transitional-flow regime, the enhancement of heat transfer was by a factor of approximately 2.5 . Friction factors for the wavy channel were about twice those for the parallel-plate channel in the steady-flow region, and remained almost constant in the transitional regime. Although some studies for steady and unsteady flows have been reported, example, Blancher et. al. (1998), Selvarajna et. al. (1998), Greiner et. al. (1991), little knowledge is available on the flow in these wavy channels. 
The analyses of the flow properties of non-Newtonian fluids are very important in the fields of fluid dynamics because of their technological application. Mechanics of non-Newtonian fluids present challenges to engineers, physicists and mathematicians. Due to the complex stress-strain relationships of non-Newtonian fluids, not many investigators have studied the flow behavior of the fluids in various flow fields. Hot rolling, extrusion of plastics, flow in journal bearings, lubrication, and flow in a shock absorber are some typical examples to name just a few (Bőhme, 1981; Huilgol et. al., 1997). Non-Newtonian fluids are of increasing importance in modern technology due to its growing use in many activities, such as molten plastic, paints, drilling, and petroleum and polymer solutions. The Walters fluid is one of such fluids.

The most commonly used technique for internal cooling enhancement is the placement of periodic ribs. Ribs are generally mounted on the heat transfer between the surfaces, which disturb the boundary layer growth and enhance the heat transfer between the surface and the fluid. In addition to ribs and impingement, a third common internal cooling enhancement technique is the placement of internal flow swirls, tape twisters, or baffles. The convective heat transfer in a vertical channel could be enhanced by using special inserts which can be specially designed to increase the included angle between the velocity vector and the temperature gradient vector rather than to promote turbulence. This increases the rate of heat transfer without a considerable drop in the pressure (Guo et. al., 1998).A plane baffle may be used as an insert to enhance the rate of heat transfer in the channel. A thin and perfectly conductive baffle is used so as to avoid a considerable increase in the transverse thermal resistance into the channel. Cheng et al. (1989) studied analytically heat transfer aspects of a laminar fully developed forced-convection within an asymmetrically heated horizontal double-passage channel and concluded that the thermal characteristics of fully developed flow could be significantly affected by the position of the baffle, the pressure gradient ratio and the thermal boundary conditions. Similar mixed convection problem in a vertical double-passage channel has been investigated analytically by Salah El-Din (1994). His results showed that the presence of the baffle may lead to a higher value of Nusselt number according to the baffle position and the value of $\mathrm{Gr} / \mathrm{Re}$. Dutta and Hossain (2005) reported experimental results of the enhancement of heat transfer with inclined solid and perforated baffles. In that study, the effects of baffle size, position were studied for internal cooling heat transfer augmentation. Chen and Chen (1998) experimental results showed that for small baffle widths, the local heat transfer coefficient decreases with an increase in the baffle wall gap. For large baffle widths, there is an optimum distance between the baffle and the solid wall which give rise to a higher heat transfer coefficient. More over recently Chang and Shiau (2005) studied numerically the effects of horizontal baffle on the heat transfer characteristics of pulsating opposing mixed convection in a vertical channel. They found that the flow pulsation with a baffle gives the optimal heat transfer. Also with large Reynolds number, the inlet flow pulsation dominates the velocity field in the channel.

The purpose of the present study focuses attention on the fully developed free convection flow of a Walter's fluid (Model B') in a vertical wavy double-passage channel in the presence of heat source. The buoyancy force, temperature ratio, heat source/sink, $\lambda x$ and viscoelastic parameter at different baffle positions are all considered so as to extensively investigate their distinct influence on the velocity, temperature, skin friction and rate of heat transfer.

\section{Mathematical formulation}

\section{Case 1: Free convection of Walter's fluid in a vertical channel with baffle}

Consider a steady two dimensional laminar free convection Walter's fluid flow in an open-ended vertical channel with one wavy wall and another flat wall as shown in Fig. 1. The channel is divided into two passages by means of a perfectly conducting thin baffle, for which the transverse thermal resistance can be neglected (Cheng et al., 1989 and Salah El Din, 1994, 2002). The $X$ axis is taken upwards and parallel to the flat wall, while the $Y$-axis is taken perpendicular to it in such a way that the wavy wall is represented by $Y=\varepsilon^{*} \cos (k X)$ and the flat wall by $Y=d$. The wavy and flat walls are maintained at a constant temperature $T_{w}$ and $T_{1}$, respectively. The following assumptions are made

a. All the fluid properties except the density in the buoyancy force are constant.

b. The dissipative effects and the work of deformation are neglected in the energy equation.

c. The wavelength of the wavy wall is large compared with the width of the channel.

d. For fully developed flow, it is assumed that the transverse velocity and temperature gradient in the axial direction are zero.

e. The volumetric heat source/sink term in the energy is constant.

f. Boussinesq approximation is assumed, i.e., $\rho=\rho_{s}\left(1-\beta\left(T-T_{s}\right)\right)$ 

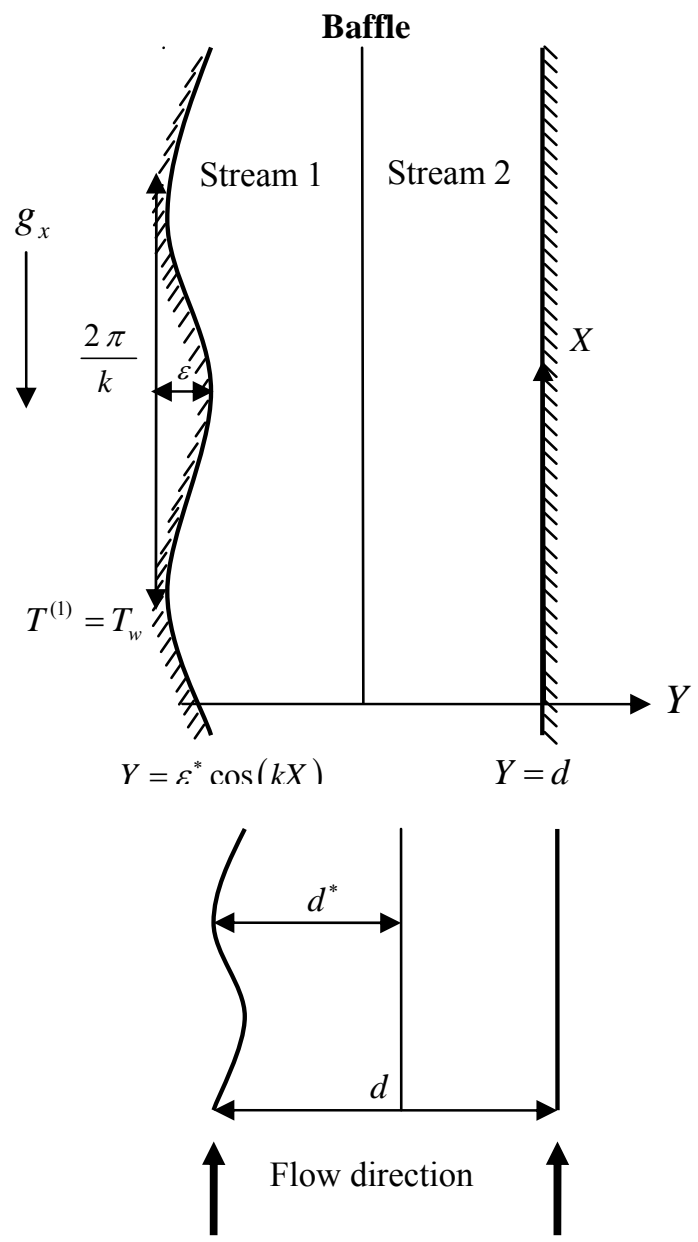

Figure.1 Physical configuration of the double-passage channel

Following the above assumptions the basic equations governing the flow are (Rita and Alok, 2000)

$$
\begin{gathered}
\frac{\partial U_{j}}{\partial X}+\frac{\partial V_{j}}{\partial Y}=0 \\
\rho\left(U_{j} \frac{\partial U_{j}}{\partial X}+V_{j} \frac{\partial U_{j}}{\partial Y}\right)=-\frac{\partial P_{j}^{*}}{\partial X}+\mu \nabla^{2} U_{j}-2 K_{0}\left(2 U_{j} \frac{\partial^{3} U_{j}}{\partial X^{3}}+2 V_{j} \frac{\partial^{3} U_{j}}{\partial X^{2} \partial Y}+U_{j} \frac{\partial^{3} U_{j}}{\partial X \partial Y^{2}}\right. \\
+U_{j} \frac{\partial^{3} V_{j}}{\partial Y \partial X^{2}}+V_{j} \frac{\partial^{3} U_{j}}{\partial Y^{3}}+V_{j} \frac{\partial^{3} V_{j}}{\partial X \partial Y^{2}}-6 \frac{\partial U_{j}}{\partial X} \frac{\partial^{2} U_{j}}{\partial X^{2}}+\frac{\partial U_{j}}{\partial Y} \frac{\partial^{2} V_{j}}{\partial X^{2}}-4 \frac{\partial U_{j}}{\partial Y} \frac{\partial^{2} U_{j}}{\partial X \partial Y}-2 \frac{\partial V_{j}}{\partial X} \frac{\partial^{2} V_{j}}{\partial Y^{2}} \\
\left.-3 \frac{\partial V_{j}}{\partial X} \frac{\partial^{2} U_{j}}{\partial X \partial Y}-\frac{\partial U_{j}}{\partial X} \frac{\partial^{2} V_{j}}{\partial X \partial Y}-3 \frac{\partial U_{j}}{\partial Y} \frac{\partial^{2} V_{j}}{\partial Y^{2}}-\frac{\partial V_{j}}{\partial X} \frac{\partial^{2} V_{j}}{\partial Y^{2}}-\frac{\partial U_{j}}{\partial X} \frac{\partial^{2} U_{j}}{\partial Y^{2}}\right)-\rho g_{X} \\
\rho\left(U_{j} \frac{\partial V_{j}}{\partial X}+V_{j} \frac{\partial V_{j}}{\partial Y}\right)=-\frac{\partial P_{j}^{*}}{\partial Y}+\mu \nabla^{2} V_{j}-2 K_{0}\left(U_{j} \frac{\partial^{3} U_{j}}{\partial X^{2} \partial Y}+U_{j} \frac{\partial^{3} V_{j}}{\partial X^{3}}+V_{j} \frac{\partial^{3} U_{j}}{\partial X \partial Y^{2}}+V_{j} \frac{\partial^{3} V_{j}}{\partial Y \partial X^{2}}\right. \\
+2 U_{j} \frac{\partial^{3} V_{j}}{\partial X \partial Y^{2}}+2 V_{j} \frac{\partial^{3} V_{j}}{\partial Y^{3}}-2 \frac{\partial U_{j}}{\partial X} \frac{\partial^{2} V_{j}}{\partial X^{2}}-2 \frac{\partial V_{j}}{\partial X} \frac{\partial^{2} V_{j}}{\partial Y^{2}}-3 \frac{\partial^{2} U_{j}}{\partial X^{2}} \frac{\partial V_{j}}{\partial X}-3 \frac{\partial V_{j}}{\partial Y} \frac{\partial^{2} U_{j}}{\partial X \partial Y}-3 \frac{\partial U_{j}}{\partial Y} \frac{\partial^{2} V_{j}}{\partial X \partial Y} \\
\left.-\frac{\partial V_{j}}{\partial Y} \frac{\partial^{2} V_{j}}{\partial X^{2}}-\frac{\partial U_{j}}{\partial Y} \frac{\partial^{2} U_{j}}{\partial X^{2}}-6 \frac{\partial V_{j}}{\partial Y} \frac{\partial^{2} V_{j}}{\partial Y^{2}}-4 \frac{\partial V_{j}}{\partial X} \frac{\partial^{2} V_{j}}{\partial X \partial Y}\right) \\
\rho C_{P}\left(U_{j} \frac{\partial T_{j}}{\partial X}+V_{j} \frac{\partial T_{j}}{\partial Y}\right)=k \nabla^{2} T_{j}+Q
\end{gathered}
$$

The boundary conditions relevant to the problem are taken as, 


$$
\begin{gathered}
U_{1}=V_{1}=0, \quad T_{1}=T_{w} \quad \text { on } Y=\varepsilon^{*} \cos (k X) \\
U_{1}=U_{2}=0, V_{1}=V_{2}=0, \quad T_{1}=T_{2}, \quad \frac{\partial T_{1}}{\partial Y}+\frac{\partial T_{1}}{\partial X}=\frac{\partial T_{2}}{\partial Y}+\frac{\partial T_{2}}{\partial X} \text { on } Y=d^{*} \\
U_{2}=V_{2}=0, \quad T_{2}=T_{f} \quad \text { on } \quad Y=d
\end{gathered}
$$

Introducing the following non-dimensional variables in the governing equations for velocity and temperature as,

$$
x=\frac{X}{d}, y=\frac{Y}{d}, u_{i}=\frac{U_{i} d}{v}, v_{i}=\frac{V_{i} d}{v}, \theta=\frac{T-T_{s}}{T_{w}-T_{s}}, \bar{p}=\frac{p^{*}}{\rho(v / d)^{2}}
$$

where $T_{s}$ is the fluid temperature in static condition. Doing this, Eqs. (1) to (5) become

$$
\begin{aligned}
& u_{j} \frac{\partial u_{j}}{\partial x}+v_{j} \frac{\partial u_{j}}{\partial y}=-\frac{\partial \overline{P_{j}}}{\partial x}+\frac{\partial^{2} u_{j}}{\partial x^{2}}+\frac{\partial^{2} u_{j}}{\partial y^{2}}-K\left(2 u_{j} \frac{\partial^{3} v_{j}}{\partial x^{3}}+2 v_{j} \frac{\partial^{3} u_{j}}{\partial x^{2} \partial y}+u_{j} \frac{\partial^{3} u_{j}}{\partial x \partial y^{2}}+u_{j} \frac{\partial^{3} v_{j}}{\partial y \partial x^{2}}\right. \\
&+v_{j} \frac{\partial^{3} u_{j}}{\partial y^{3}}+v_{j} \frac{\partial^{3} v_{j}}{\partial x \partial y^{2}}-6 \frac{\partial u_{j}}{\partial x} \frac{\partial^{2} u_{j}}{\partial x^{2}}+\frac{\partial u_{j}}{\partial y} \frac{\partial^{2} v_{j}}{\partial x^{2}}-4 \frac{\partial u_{j}}{\partial y} \frac{\partial^{2} u_{j}}{\partial x \partial y}-2 \frac{\partial v_{j}}{\partial x} \frac{\partial^{2} v_{j}}{\partial y^{2}} \\
&\left.-3 \frac{\partial v_{j}}{\partial x} \frac{\partial^{2} u_{j}}{\partial x \partial y}-\frac{\partial u_{j}}{\partial x} \frac{\partial^{2} v_{j}}{\partial x \partial y}-3 \frac{\partial u_{j}}{\partial y} \frac{\partial^{2} v_{j}}{\partial y^{2}}-\frac{\partial v_{j}}{\partial x} \frac{\partial^{2} v_{j}}{\partial y^{2}}-\frac{\partial u_{j}}{\partial x} \frac{\partial^{2} u_{j}}{\partial y^{2}}\right)-G \theta_{j} \\
& u_{j} \frac{\partial v_{j}}{\partial x}+v_{j} \frac{\partial v_{j}}{\partial y}=-\frac{\partial \overline{P_{j}}}{\partial y}+\frac{\partial^{2} v_{j}}{\partial x^{2}}+\frac{\partial^{2} v_{j}}{\partial y^{2}}-K\left(u_{j} \frac{\partial^{3} u_{j}}{\partial x^{2} \partial y}+u_{j} \frac{\partial^{3} v_{j}}{\partial x^{3}}+v_{j} \frac{\partial^{3} u_{j}}{\partial x \partial y^{2}}+v_{j} \frac{\partial^{3} v_{j}}{\partial y \partial x^{2}}\right. \\
&+ 2 u_{j} \frac{\partial^{3} v_{j}}{\partial x \partial y^{2}}+2 v_{j} \frac{\partial^{3} v_{j}}{\partial y^{3}}-2 \frac{\partial u_{j}}{\partial x} \frac{\partial^{2} v_{j}}{\partial x^{2}}-2 \frac{\partial v_{j}}{\partial x} \frac{\partial^{2} v_{j}}{\partial y^{2}}-3 \frac{\partial^{2} u_{j}}{\partial x^{2}} \frac{\partial v_{j}}{\partial x}-3 \frac{\partial v_{j}}{\partial y} \frac{\partial^{2} u_{j}}{\partial x \partial y} \\
&\left.-3 \frac{\partial u_{j}}{\partial y} \frac{\partial^{2} v_{j}}{\partial x \partial y}-\frac{\partial v_{j}}{\partial y} \frac{\partial^{2} v_{j}}{\partial x^{2}}-\frac{\partial u_{j}}{\partial y} \frac{\partial^{2} u_{j}}{\partial x^{2}}-6 \frac{\partial v_{j}}{\partial y} \frac{\partial^{2} v_{j}}{\partial y^{2}}-4 \frac{\partial_{j}}{\partial x} \frac{\partial^{2} v_{j}}{\partial x \partial y}\right) \\
& P\left(u_{j} \frac{\partial \theta_{j}}{\partial x}+v_{j} \frac{\partial \theta_{j}}{\partial y}\right)=\frac{\partial^{2} \theta_{j}}{\partial x^{2}}+\frac{\partial^{2} \theta_{j}}{\partial y^{2}}+\alpha
\end{aligned}
$$

Subject to the boundary conditions,

where

$$
\begin{array}{ll}
P=\eta_{0} c_{p} / k, & \text { the Prandtl number } \\
\varepsilon=\varepsilon^{*} / d, & \text { the dimensionless amplitude parameter } \\
\lambda=k d, & \text { the dimensionless frequency parameter } \\
m=\left(T_{1}-T_{s}\right) /\left(T_{w}-T_{s}\right), & \text { the wall temperature ratio } \\
\alpha=Q d^{2} / k\left(T_{w}-T_{s}\right), & \text { the dimensionless heat source/sink parameter } \\
K=2 K_{0} /\left(\rho d^{2}\right), & \text { the dimensionless viscoelastic parameter } \\
G=\frac{d^{3} g_{x} \beta\left(T_{w}-T_{s}\right)}{v^{2}}, & \text { the Grashof number }
\end{array}
$$

where the subscript $S$ denotes quantities in the static fluid condition. 
The term $\eta_{0}$ is the limiting viscosity at small rate of shear which is given by

$$
\eta_{0}=\int_{0}^{\infty} N(\tau) d \tau \text { and } k_{0}=\int_{0}^{\infty} \tau N(\tau) d \tau
$$

where $N(\tau)$ being the relaxation spectrum as introduced by Walters $(1960,1962)$. This idealized model is a valid approximation of Walter's fluid (model $B^{\prime}$ ) taking very short memories into account so that terms involving

$$
\int_{0}^{\infty} \tau^{n} N(\tau) d \tau, n \geq 2
$$

are neglected (Rita and Alok, 2000).

\section{Solutions}

Equations (8) to (10) are coupled non-linear partial differential equations and hence finding exact solutions is out of scope. However, for small values of the amplitude parameter $\varepsilon$, approximate solutions can be extracted through the perturbation method. The amplitude parameter $\varepsilon$ is usually small and hence regular perturbation method can be strongly justified. Adopting this technique, solutions for velocity and temperature are assumed in the form

$$
\begin{aligned}
& u_{j}(x, y)=u_{j 0}(y)+u_{j 1}(x, y) \quad v_{j}(x, y)=v_{j 1}(x, y) \\
& \bar{p}_{j}=p_{j 0}(y)+p_{j 1}(x, y), \theta_{j}(x, y)=\theta_{j 0}(y)+\theta_{j 1}(x, y)
\end{aligned}
$$

where the perturbations $u_{j 1}, v_{j 1}, p_{j 1}$ and $\theta_{j 1}$ are small compared with the mean or zeroth order quantities. Equations (7) to (11) yield the following equations.

Zeroth order equations

$$
\frac{d^{2} u_{j 0}}{d y^{2}}+G \theta_{j 0}=0 \quad \frac{d^{2} \theta_{j 0}}{d y^{2}}=-\alpha
$$

First order equations

$$
\begin{aligned}
& u_{j 0} \frac{\partial u_{j 1}}{\partial x}+v_{j 1} \frac{\partial u_{j 0}}{\partial y}=-\frac{\partial \overline{P_{j 1}}}{\partial x}+\frac{\partial^{2} u_{j 1}}{\partial x^{2}}+\frac{\partial^{2} u_{j 1}}{\partial y^{2}}-K\left(2 u_{j 0} \frac{\partial^{3} u_{j 1}}{\partial x^{3}}+u_{j 0} \frac{\partial v_{j 1}}{\partial x}=0\right. \\
& \left.+v_{j 1} \frac{\partial^{3} u_{j 0}}{\partial y^{3}}+\frac{\partial u_{j 0}}{\partial y} \frac{\partial^{2} v_{j 1}}{\partial x^{2}}-4 \frac{\partial u_{j 0}}{\partial y} \frac{\partial^{2} u_{j 1}}{\partial x \partial y}-2 \frac{\partial v_{j 1}}{\partial y} \frac{\partial^{2} u_{j 0}}{\partial y^{2}}-3 \frac{\partial u_{j 0}}{\partial y} \frac{\partial^{2} v_{j 1}}{\partial y^{2}}-\frac{\partial u_{j 1}}{\partial x} \frac{\partial^{2} u_{j 0}}{\partial y^{2}}\right)-G \theta_{j 1} \\
& u_{j 0} \frac{\partial v_{j 1}}{\partial x}=-\frac{\partial \overline{P_{j 1}}}{\partial y}+\frac{\partial^{2} v_{j 1}}{\partial x^{2}}+\frac{\partial^{2} v_{j 1}}{\partial y^{2}}-K\left(u_{j 0} \frac{\partial^{3} u_{j 1}}{\partial x^{2} \partial y}+2 u_{j 0} \frac{\partial^{3} v_{j 1}}{\partial x \partial y^{2}}+u_{j 0} \frac{\partial^{3} v_{j 1}}{\partial x^{3}}-2 \frac{\partial v_{j 1}}{\partial x} \frac{\partial^{2} u_{j 0}}{\partial x^{2}}\right. \\
& \left.-3 \frac{\partial^{2} v_{j 1}}{\partial x \partial y}-\frac{\partial u_{j 0}}{\partial y} \frac{\partial^{2} u_{j 1}}{\partial x^{2}}\right) \quad P\left(u_{j 0} \frac{\partial \theta_{j 1}}{\partial x}+v_{j 1} \frac{d \theta_{j 0}}{d y}\right)=\frac{\partial^{2} \theta_{j 1}}{\partial x^{2}}+\frac{\partial^{2} \theta_{j 1}}{\partial y^{2}}
\end{aligned}
$$

In deriving the Eq. (13), the constant pressure gradient term $\frac{\partial}{\partial x}\left(p_{0}-p_{s}\right)$ has been taken equal to zero following (Ostrach, 1952). In view of Eq. (12) the boundary condition in Eq. (11) can be split up into the following two parts.

Zeroth order boundary conditions

First order boundary conditions

$$
\begin{aligned}
& u_{10}=0, \quad \theta_{10}=1 \quad \text { on } y=0 \\
& u_{10}=u_{20}=0, \theta_{10}=\theta_{20}, \frac{d \theta_{10}}{d y}=\frac{d \theta_{20}}{d y} \text { on } y=y^{*} \\
& u_{20}=0, \theta_{20}=m \text { on } \quad y=1
\end{aligned}
$$




$$
\begin{aligned}
& u_{11}=-r p\left(e^{i \lambda x} \frac{d u_{10}}{d y}\right), \quad v_{11}=0, \theta_{11}=-r p\left(e^{i \lambda x} \frac{d \theta_{10}}{d y}\right) \text { on } y=0 \\
& u_{11}=u_{21}=0, \quad v_{11}=v_{21}=0, \theta_{11}=\theta_{21}, \text { on } y=y^{*} \\
& u_{21}=0, \quad v_{21}=0, \theta_{21}=0, \text { on } \quad y=1
\end{aligned}
$$

where $r p$ represents the real part

The solutions for zeroth order velocity $u_{j 0}$ and zeroth order temperature $\theta_{j 0}$ satisfying the Eq. (13) and the boundary conditions Eq. (17) are given by

Stream 1

Stream 2

$$
\begin{gathered}
\theta_{10}=\frac{-\alpha y^{2}}{2}+c_{1} y+c_{2} \\
u_{10}=l_{1} y^{4}+l_{2} y^{3}+l_{3} y^{2}+d_{1} y+d_{2}
\end{gathered}
$$

$$
\begin{gathered}
\theta_{20}=\frac{-\alpha y^{2}}{2}+c_{7} y+c_{8} \\
u_{20}=l_{7} y^{4}+l_{8} y^{3}+l_{9} y^{2}+d_{11} y+d_{12}
\end{gathered}
$$

In order to solve Eqs. (14) to (17) for the first order quantity it is convenient to introduce stream function $\bar{\psi}$ in the following form

$$
u_{j 1}=-\frac{\partial \bar{\psi}_{j 1}}{\partial y}, v_{j 1}=\frac{\partial \bar{\psi}_{j 1}}{\partial x} \text { for } j=1,2
$$

The stream function approach reduces the number of dependent variables to be solved and also eliminates pressure from the list of variables. Differentiate Eq. (15) with respect to $y$ and differentiate Eq. (16) with respect to $x$ and then subtract Eq. (15) with Eq. (16) which will result in the elimination of pressure $p_{i 1}$. We assume stream function and temperature in the following form

$$
\bar{\psi}_{j 1}(x, y)=\varepsilon e^{i \lambda x} \psi_{j 1}(y), \quad \theta_{j 1}(x, y)=\varepsilon e^{i \lambda x} t_{j 1}(y)
$$

From the above Eqs. (14) to (17) after elimination of $P_{j 1}$, can be expressed in terms of the stream function $\psi$ and $t$ in the form

$$
\begin{gathered}
\psi_{j}^{i v}-\psi_{j}^{\prime \prime}\left(2 \lambda^{2}+i \lambda u_{j 0}\right)+\psi_{j}\left(\lambda^{4}+i \lambda u_{j 0}^{\prime \prime}+i u_{j 0} \lambda^{3}\right)+K i\left(2 u_{j 0} \lambda^{3} \psi_{j}^{\prime \prime}\right. \\
\left.-\lambda u_{j 0} \psi_{j}^{i v}-2 u_{j 0}^{\prime} \lambda^{3} \psi_{j}^{\prime}-3 u_{j 0}^{\prime \prime} \lambda^{3} \psi_{j}+\lambda u_{10}^{i v} \psi_{j}-\lambda^{5} u_{10} \psi_{j}\right)=G t_{j}^{\prime} \\
t_{j}^{\prime \prime}-\lambda^{2} t_{j}=\operatorname{Pi} \lambda\left(u_{10} t_{j}+\psi_{j} \theta_{j 0}^{\prime}\right)
\end{gathered}
$$

where $i$ is the coefficient of imaginary part and suffix $(j=1,2)$ denotes stream- 1 and stream-2 respectively. Boundary conditions as defined in Eq. (19) can be written in terms of $\psi$ and $t$ as

$$
\begin{aligned}
& \psi_{1}^{\prime}=u_{10}^{\prime}, \quad \psi_{1}=0, \quad t_{1}=-\theta_{10}^{\prime} \quad \text { on } \quad y=0 \\
& \psi_{1}^{\prime}=0, \quad \psi_{1}=0, \quad \psi_{2}^{\prime}=0, \quad \psi_{2}=0, \text { on } y=y^{*} \\
& t_{1}=t_{2}, \quad t_{1}^{\prime}=t_{2}^{\prime}, \quad \text { on } y=y^{*} \\
& \psi_{2}^{\prime}=0, \quad \psi_{2}=0, \quad t_{2}=0, \quad \text { on } \quad y=1
\end{aligned}
$$

We restrict our attention to the real parts of the solutions for the perturbed quantities $\psi, t, u_{j 1}$ and $v_{j 1}$. Consider only small values of $\lambda$. On substituting

$$
\psi(\lambda, y)=\sum_{r=0}^{\infty} \lambda^{r} \psi_{r}, t(\lambda, y)=\sum_{r=0}^{\infty} \lambda^{r} t_{r}
$$

into Eqs. (25) to (27) we obtain to the order of $\lambda$, the following set of ordinary differential equations

Zeroth order

First order

$$
\begin{aligned}
t_{i 0}^{\prime \prime} & =0 \\
\psi_{j 0}^{i v} & =G t_{j 0}^{\prime}
\end{aligned}
$$

$$
\begin{gathered}
t_{i 1}^{\prime \prime}=P i\left(u_{j 0} t_{j 0}+\psi_{j 0} \theta_{j 0}^{\prime}\right) \\
\psi_{j 1}^{i v}=i u_{j 0} \psi_{j 0}^{\prime \prime}-i u_{j 0}^{\prime \prime} \psi_{j 0}+i K\left(u_{j 0} \psi_{j 0}^{i v}-u_{j 0}^{i v} \psi_{j 0}\right)+G t_{j 1}^{\prime}
\end{gathered}
$$


Zeroth order boundary conditions in terms of stream function and temperature are

$$
\begin{aligned}
& \psi_{10}^{\prime}=u_{10}^{\prime}, \quad \psi_{10}=0, t_{10}=-\theta_{10}^{\prime} \quad \text { on } y=0 \\
& \psi_{10}^{\prime}=0, \psi_{10}=0, \psi_{20}^{\prime}=0, \psi_{20}=0, t_{10}=t_{20}, t_{10}^{\prime}=t_{20}^{\prime}, \text { on } \quad y=y^{*} \\
& \psi_{20}^{\prime}=0, \psi_{20}=0, t_{20}=0, \text { on } \quad y=1
\end{aligned}
$$

First order boundary conditions in terms of stream function and temperature are

$$
\begin{aligned}
& \psi_{11}^{\prime}=0, \psi_{11}=0, t_{11}=0 \quad \text { on } \quad y=0 \\
& \psi_{11}^{\prime}=0, \psi_{11}=0, \psi_{21}^{\prime}=0, \psi_{21}=0, \text { on } y=y^{*} \\
& t_{11}=t_{21}, t_{11}^{\prime}=t_{21}^{\prime}, \text { on } y=y^{*} \\
& \psi_{21}^{\prime}=0, \psi_{21}=0, t_{21}=0, \text { on } \quad y=1
\end{aligned}
$$

The set of Eqs. (25) to (28) subject to boundary conditions Eqs. (29) and (30) have been solved exactly for $\psi$ and $t$. From these solutions, the first order quantities can be put in the form,

$$
\psi=\left(\psi_{r p}+\mathrm{i} \psi_{i p}\right)=\psi_{j 0}+\lambda \psi_{j 1}, \quad t=\left(t_{r p}+\mathrm{i} t_{i p}\right)_{j}=t_{j 0}+\lambda t_{j 1}
$$

where suffix $r p$ denotes the real part and ip denotes the imaginary part. Considering only the real part, the expression for first order velocity and temperature become

$$
\begin{gathered}
u_{j 1}=\varepsilon\left(-\cos (\lambda x) \psi_{j 0}^{\prime}+\lambda \psi_{j 1}^{\prime} \sin (\lambda x)\right) \\
v_{j 1}=\varepsilon\left(-\lambda^{2} \cos (\lambda x) \psi_{j 1}-\lambda \psi_{j 0} \sin (\lambda x)\right) \\
\theta_{j 1}=\varepsilon\left(\cos (\lambda x)\left(t_{j 0}\right)-\lambda t_{j 1} \sin (\lambda x)\right)
\end{gathered}
$$

The first order and total solutions are given in Appendix.

\subsection{Skin friction and Nusselt number}

The shearing stress $\sigma_{x y}$ at any point in the fluid in non-dimensional form is given by

$$
\begin{aligned}
& \sigma_{x y}=\frac{d^{2} \bar{\sigma}_{x y}}{\rho v^{2}}=u_{0}^{\prime}(y)+\varepsilon e^{i \lambda x} \bar{u}_{1}^{\prime}(y)+i \varepsilon \lambda e^{i \lambda x} \bar{v}_{1}(y)+K \varepsilon\left(3 u_{0}^{\prime} e^{i \lambda x} \bar{v}_{1}^{\prime}(y)+u_{0}^{\prime}(i \lambda) e^{i \lambda x} \bar{u}_{1}(y)\right. \\
& \left.-u_{0}^{\prime \prime}(y) e^{i \lambda x} \bar{v}_{1}(y)+u_{0}(y) \lambda^{2} e^{i \lambda x} \bar{v}_{1}(y) u_{0}(y)(i \lambda) e^{i \lambda x} \bar{u}_{1}^{\prime}(y)\right)
\end{aligned}
$$

At the wavy wall, $y=\varepsilon \cos (\lambda x)$ skin friction takes the form

$$
\begin{aligned}
& \sigma_{w}=\sigma_{0}^{0}(0)+\varepsilon\left(u_{10}^{\prime \prime} \cos (\lambda x)+\lambda \psi_{11}^{\prime \prime}(0) \sin (\lambda x)-\psi_{10}^{\prime \prime}(0) \cos (\lambda x)\right) \\
& -2 \lambda \varepsilon K \sigma_{0}^{0}(0)\left(\psi_{10}^{\prime}(0) \sin (\lambda x)+\lambda \psi_{11}^{\prime}(0) \cos (\lambda x)\right)
\end{aligned}
$$

and at the flat wall, $y=1$ skin friction takes the form

$$
\begin{aligned}
\sigma_{f} & =\sigma_{1}^{0}(0)+\varepsilon\left(-\psi_{20}^{\prime \prime}(1) \cos (\lambda x)+\lambda \psi_{21}^{\prime \prime}(1) \sin (\lambda x)\right) \\
& -\varepsilon \lambda K\left(\lambda u_{20}^{\prime \prime}(1) \psi_{21}(1) \cos (\lambda x)-2 u_{20}^{\prime}(1)\left(\psi_{21}^{\prime}(1) \sin (\lambda x)+\lambda \psi_{21}^{\prime} \cos (\lambda x)\right)\right)
\end{aligned}
$$

where $\sigma_{0}^{0}(0)=\left(\frac{d u_{10}}{d y}\right)_{y=0}$ and $\sigma_{1}^{0}(1)=\left(\frac{d u_{20}}{d y}\right)_{y=1}$ are the zeroth order skin-friction at the walls, and $\bar{u}_{1}(y)$ and $\bar{v}_{1}(y)$ are given by

$$
u(x, y)=e^{i \lambda x} \bar{u}_{1}(y), v_{1}(x, y)=e^{i \lambda x} \bar{v}_{1}(y)
$$

The non-dimensional heat transfer coefficient known as Nusselt number $(\mathrm{Nu})$ is given by

$$
N u=\frac{\partial \theta}{\partial y}=\theta_{0}^{\prime}(y)+\varepsilon \operatorname{Re}\left(e^{i \lambda x} \theta_{1}^{\prime}(y)\right)
$$

At the wavy wall $y=-1+\varepsilon \cos (\lambda x)$ Nusselt number $N u_{w}$ takes the form

$$
N u_{w}=N u_{0}^{0}(0)+\varepsilon\left(\theta_{10}^{\prime}(0) \cos (\lambda x)+t_{10}^{\prime}(0) \cos (\lambda x)-\lambda t_{11}^{\prime}(0) \sin (\lambda x)\right)
$$


and at the flat wall $y=1$,

$$
N u_{f}=N u_{1}^{0}(1)+\varepsilon\left(t_{20}^{\prime}(1) \cos (\lambda x)-\lambda t_{21}^{\prime}(1) \sin (\lambda x)\right)
$$

where $N u_{0}^{0}(0)=\left(\frac{d \theta_{10}}{d y}\right)_{y=0}$ and $N u_{1}^{0}(1)=\left(\frac{d \theta_{20}}{d y}\right)_{y=1}$ are zeroth order Nusselt number at the walls.

Velocity and temperature solutions are numerically evaluated for several sets of values of the governing parameters. Also, the wall skin friction $\sigma_{w}, \sigma_{f}$ and the wall Nusselt number $N u_{w}, N u_{f}$ are calculated numerically and some of the qualitative interesting features are presented.

\section{Case 2a: Comparison of the Solutions with Salah El Din (2002) in the presence of baffle}

To validate the results of the present model, the problem is solved in the absence of viscoelastic parameter, product of nondimensional wave number and space co-ordinate, pressure gradient and heat source. The dimensionless basic equations (7) to (10) in the absence of the above parameters become

$$
\begin{gathered}
\frac{d^{2} \theta_{i 0}}{d y^{2}}=-\alpha \\
\frac{d^{2} u_{j 0}}{d y^{2}}+G \theta_{j 0}=0
\end{gathered}
$$

To compare the results, the boundary conditions on temperature are taken as in Salah El Din (2002), i.e..,

$$
\begin{aligned}
& \theta_{1}=-\frac{1}{2} \quad \text { on } y=0 ; \quad \theta_{2}=\frac{1}{2} \quad \text { on } y=1 \\
& \theta_{1}=\theta_{2}, \frac{d \theta_{1}}{d y}=\frac{d \theta_{2}}{d y} \text { on } y=y^{*}
\end{aligned}
$$

The boundary conditions on velocity are no-slip conditions on the boundary and vanishing of velocity at the baffle. That is

$$
\begin{aligned}
& u_{1}=0 \quad \text { on } y=0 ; \quad u_{2}=0 \quad \text { on } y=1 \\
& u_{1}=u_{2}=0 \text { on } y=y^{*}
\end{aligned}
$$

The solutions of Eqs. (45) and (46) using boundary conditions (47) and (48) become

$$
\begin{aligned}
& \theta_{1}=y-\frac{1}{2}, u_{1}=-\frac{G}{2}\left(\frac{y^{3}}{3}-\frac{y^{2}}{2}\right)+d_{1} y+d_{2} \\
& \theta_{2}=y-\frac{1}{2}, u_{2}=-\frac{G}{2}\left(\frac{y^{3}}{3}-\frac{y^{2}}{2}\right)+d_{3} y+d_{4}
\end{aligned}
$$

The above solutions agree very well with the solutions of Salah El Din (2002)

\section{Case 2b: Comparision of the Solutions with Rita and Alok (2000) in the absence of baffle}

The above case validates the results for viscous fluid. To validate the results for Walter's fluid, the problem is solved in the absence of the baffle and compared the results with Rita and Alok (2000). The comparison of the present model is carried out in two ways,

1. Shifting the baffle to the right wall and comparing the solutions of stream-1 with Rita and Alok (2000).

The boundary conditions for this case become

$$
\begin{array}{lll}
\theta_{10}=1 \quad \text { on } y=0 & \theta_{10}=m \quad \text { on } y=1 \\
u_{10}=0 & \text { on } y=0 & u_{10}=0 \quad \text { on } y=1 \\
t_{10}=-\theta_{10}^{\prime} \text { on } y=0 & t_{10}=0 \quad \text { on } y=1 \\
\psi_{10}^{\prime}=u_{10}^{\prime}, \psi_{10}=0 \text { on } y=0 & \psi_{10}^{\prime}=0, \psi_{10}=0 \quad \text { on } y=1 \\
t_{11}=0 & \text { on } y=0 & t_{11}=0 \quad \text { on } y=1 \\
\psi_{11}^{\prime}=0, \psi_{11}=0 \text { on } y=0 & \psi_{11}^{\prime}=0, \psi_{11}=0 \text { on } y=1
\end{array}
$$

The solution of stream-1 with above boundary conditions become

$$
\theta_{10}=\frac{-\alpha y^{2}}{2}+c_{1} y+c_{2}
$$




$$
\begin{gathered}
u_{10}=l_{1} y^{4}+l_{2} y^{3}+l_{3} y^{2}+d_{1} y+d_{2} \\
t_{10}=c_{3} y+c_{4} \\
\psi_{10}=l_{4} y^{4}+\frac{d_{3}}{6} y^{3}+\frac{d_{4}}{2} y^{2}+d_{5} y+d_{6} \\
t_{11}=P i\left(m_{1} y^{7}+m_{2} y^{6}+m_{3} y^{5}+m_{4} y^{4}+m_{5} y^{3}+m_{6} y^{2}\right)+c_{5} y+c_{6} \\
\psi_{11}=i\left(\frac{n_{1}}{5040} y+\frac{n_{2}}{3024} y^{9}+\frac{n_{3}}{1680} y^{8}+\frac{n_{4}}{840} y^{7}+\frac{n_{5}}{360} y^{6}+\frac{n_{6}}{120} y^{5}+\frac{n_{7}}{24} y^{4}\right)+\frac{n_{8}}{24} y^{4}+\frac{d_{7}}{6} y^{3} \\
+\frac{d_{8}}{2} y^{2}+d_{9} y+d_{10}
\end{gathered}
$$

With the above solutions the velocity and temperature field become

$$
\begin{gathered}
u_{1}=u_{10}+\varepsilon\left(-\cos (\lambda x) \psi_{10}^{\prime}+\lambda \psi_{11}^{\prime} \sin (\lambda x)\right) \\
v_{1}=\varepsilon\left(-\lambda^{2} \cos (\lambda x) \psi_{11}-\lambda \psi_{10} \sin (\lambda x)\right) \\
\theta_{1}=\theta_{10}+\varepsilon\left(\cos (\lambda x)\left(t_{10}\right)-\lambda t_{11} \sin (\lambda x)\right)
\end{gathered}
$$

Equations (52) to (54) are computed and are tabulated in Table 5. The above solutions agree very well with Rita and Alok (2000)

2. Shifting the baffle to the left wall and comparing the solutions of stream-2 with Rita and Alok (2000).

The boundary conditions for this case become

$$
\begin{aligned}
& \theta_{20}=0 \quad \text { on } y=0 \\
& u_{20}=0 \text { on } y=0 \\
& \theta_{20}=m \quad \text { on } \quad y=1 \\
& t_{20}=-\theta_{10}^{\prime} \text { on } y=0 \\
& u_{20}=0 \text { on } y=1 \\
& \psi_{20}^{\prime}=u_{20}^{\prime}, \psi_{20}=0 \text { on } y=0 \\
& t_{20}=0 \quad \text { on } y=1 \\
& t_{21}=0 \quad \text { on } y=0 \\
& \psi_{20}^{\prime}=0, \psi_{20}=0 \text { on } y=1 \\
& \psi_{21}^{\prime}=0, \psi_{21}=0 \text { on } y=0 \\
& t_{21}=0 \\
& \text { on } y=1 \\
& \psi_{21}^{\prime}=0, \psi_{21}=0 \text { on } y=1
\end{aligned}
$$

The solutions of stream-2 with above boundary conditions become

$$
\begin{gathered}
\theta_{20}=\frac{-\alpha y^{2}}{2}+c_{7} y+c_{8} \\
u_{20}=l_{7} y^{4}+l_{8} y^{3}+l_{9} y^{2}+d_{11} y+d_{12} \\
t_{20}=c_{9} y+c_{10} \\
\psi_{20}=l_{10} y^{4}+\frac{d_{13}}{6} y^{3}+\frac{d_{14}}{2} y^{2}+d_{15} y+d_{16} \\
t_{21}=P i\left(m_{7} y^{7}+m_{8} y^{6}+m_{9} y^{5}+m_{10} y^{4}+m_{11} y^{3}+m_{12} y^{2}\right)+c_{11} y+c_{12} \\
\left.+\frac{d_{18}}{2} y^{2}+d_{19} y+d_{20} y^{10}+\frac{n_{12}}{3024} y^{9}+\frac{n_{13}}{1680} y^{8}+\frac{n_{14}}{840} y^{7}+\frac{n_{15}}{360} y^{6}+\frac{n_{16}}{120} y^{5}+\frac{n_{17}}{24} y^{4}\right)+\frac{n_{18}}{24} y^{4}+\frac{d_{17}}{6} y^{3}
\end{gathered}
$$

With the above solutions the velocity temperature filed become

$$
\begin{gathered}
u_{2}=u_{20}+\varepsilon\left(-\cos (\lambda x) \psi_{20}^{\prime}+\lambda \psi_{21}^{\prime} \sin (\lambda x)\right) \\
v_{2}=\varepsilon\left(-\lambda^{2} \cos (\lambda x) \psi_{21}-\lambda \psi_{20} \sin (\lambda x)\right) \\
\theta_{2}=\theta_{20}+\varepsilon\left(\cos (\lambda x)\left(t_{20}\right)-\lambda t_{21} \sin (\lambda x)\right)
\end{gathered}
$$

Equations (56) to (58) are computed and are tabulated in Table 5. These solutions agree very well with Rita and Alok (2000). The constants are defined in the Appendix section

\section{Results and discussion}

The objective of the present study is to examine the characteristics of free convection of Walter's fluid (model B') in a vertical channel one of whose wall is wavy, containing a thin conducting baffle in the presence of heat source. Non -linear partial 
differential equations governing the motion has been solved by linearization technique wherein, the flow is assumed to consist to be in two parts; a mean part and a perturbed part. Exact solutions are obtained for the mean part and the perturbed part using long wave approximation. Prandtl number, wave number, amplitude parameters are fixed as $0.7,0.01,0.1$ respectively for all computations, whereas, Grashof number, wall temperature ratio, internal heat source, viscoelastic parameter and $\lambda x$ are fixed as $20,1.0,2,0$ and 1.57079632 respectively for all the graphs except the varying one.

The effect of Grashof number $G$ on the main and cross velocities are shown in Figures 2 and 3 at three different baffle positions $\left(y^{*}=0.2,0.5\right.$ and 0.8$)$, keeping wavy and flat walls at equal temperatures. As the Grashof number $G$ increases main velocity $u$ increases in both the streams at different positions of the baffle. Since the wall temperatures at both the walls are equal, the maximum point of the velocity profiles is equal in both the streams when the baffle is positioned in the centre of the channel. However when the baffle is placed near the left wall, the maximum point of velocity is in stream-2 and when the baffle is placed near the right wall, the maximum point of velocity is in stream-1 as seen in Figure 2. Irrespective of the baffle position, velocity increases as Grashof number $G$ increases, because as $G$ increases both the velocity and temperature fields are enhanced. Physically, an increase in Grashof number means an increase of the buoyancy force, which supports the motion. Similar result is found in the single passage flow examined by Aung and Worku (1986) for flat wall.

The effect of Grashof number $G$ on cross velocity as shown in Figure 3 is exactly opposite to its effect on main velocity. As $G$ increases cross velocity decreases in both the streams at any position of the baffle. Also the minimum point of cross velocity is in stream-1 only at any position of the baffle. The effect of Grashof number $G$ on temperature is shown in Table-1. It is noticed that the temperature remains invariant up to $10^{-3}$.
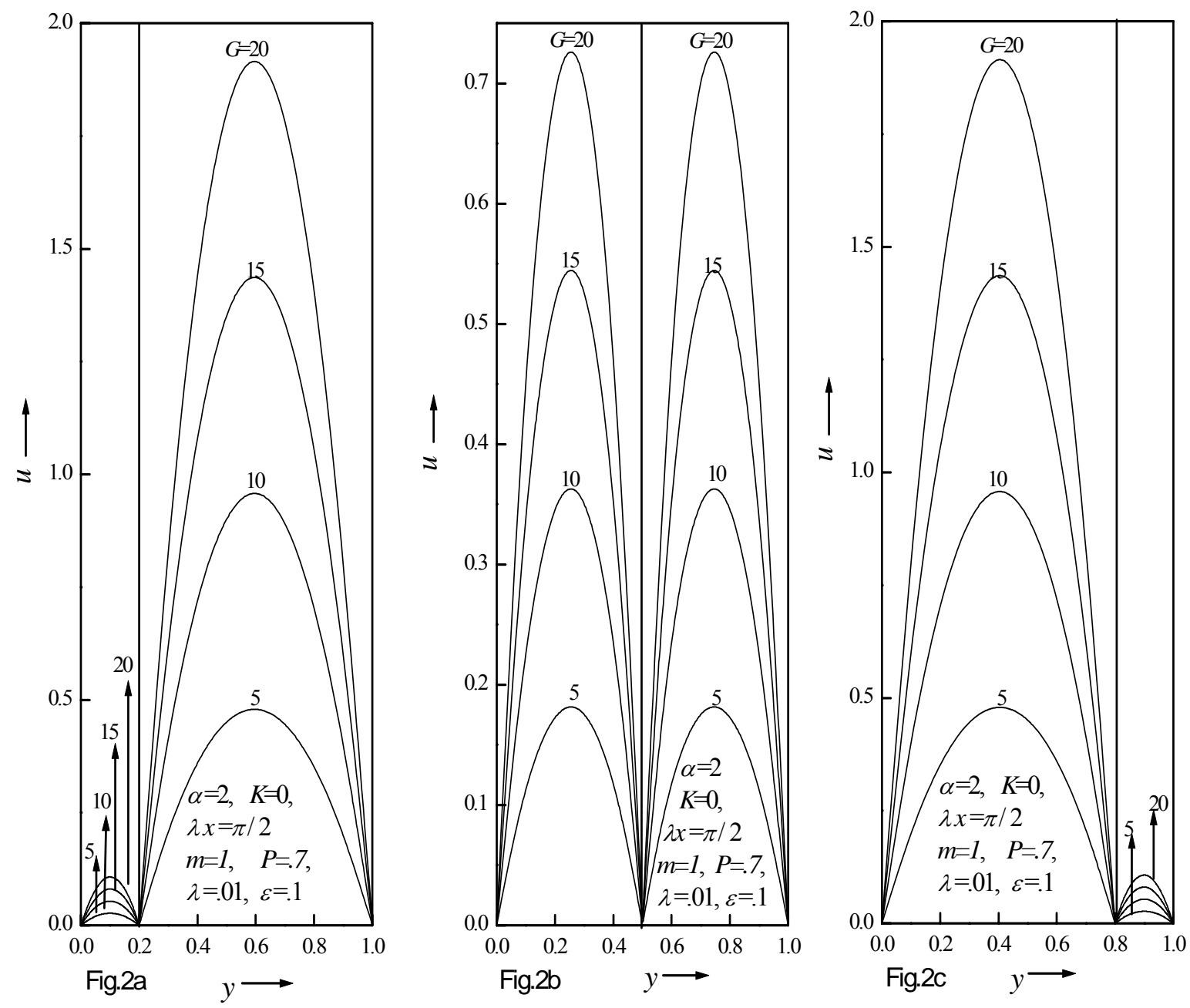

Figure 2: Main velocity profiles for differnt values of Grashof number $G$ 

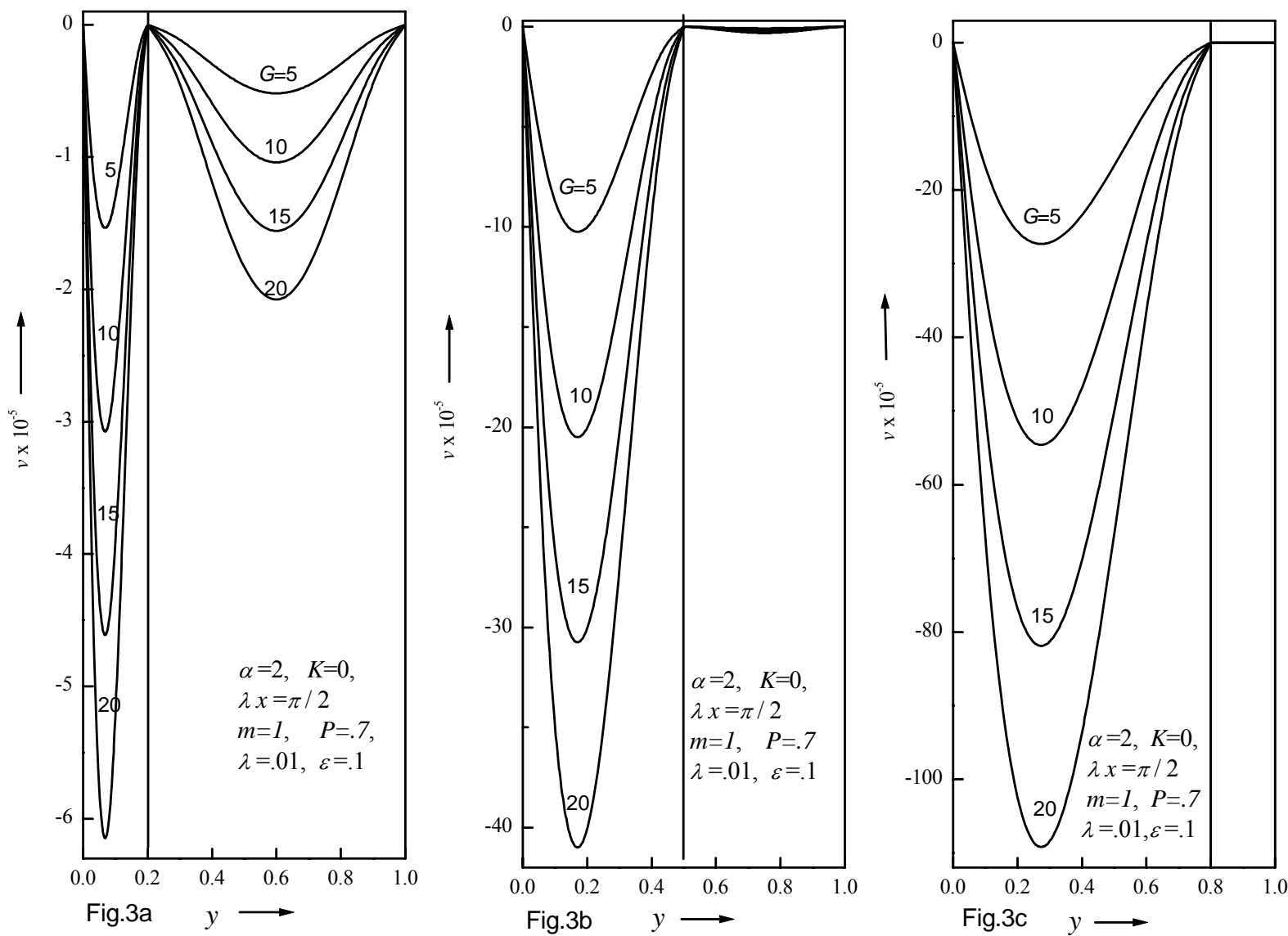

Figure 3: Cross velocity profiles for differnt values of Grashof number $G$

Table 1. Temperature values for different Grashof number and baffle position.

\begin{tabular}{|c|c|c|c|c|c|c|c|c|}
\hline \multirow{2}{*}{$\mathrm{y}$} & \multicolumn{2}{|c|}{$y^{*}=0.2$} & \multirow{2}{*}{$\mathrm{y}$} & \multicolumn{2}{|c|}{$y^{*}=0.5$} & \multirow{2}{*}{$\mathrm{y}$} & \multicolumn{2}{|c|}{$y^{*}=0.8$} \\
\cline { 2 - 5 } \cline { 5 - 6 } & $\mathrm{G}=10$ & $\mathrm{G}=200$ & & $\mathrm{G}=10$ & $\mathrm{G}=200$ & & $\mathrm{G}=10$ & $\mathrm{G}=200$ \\
\hline 0 & 1 & 1 & 0 & 1 & 1 & 0 & 1 & 1 \\
\hline 0.1 & 1.08999 & 1.08986 & 0.1 & 1.09 & 1.08993 & 0.1 & 1.08999 & 1.08982 \\
\hline 0.2 & 1.15999 & 1.15972 & 0.2 & 1.15999 & 1.15987 & 0.2 & 1.15998 & 1.15966 \\
\hline 0.2 & 1.15999 & 1.15972 & 0.3 & 1.20999 & 1.20983 & 0.3 & 1.20998 & 1.20953 \\
\hline 0.3 & 1.20998 & 1.20958 & 0.4 & 1.23999 & 1.23982 & 0.4 & 1.23997 & 1.23946 \\
\hline 0.4 & 1.23997 & 1.23949 & 0.5 & 1.24999 & 1.24983 & 0.5 & 1.24997 & 1.24946 \\
\hline 0.5 & 1.24997 & 1.24946 & 0.5 & 1.24999 & 1.24983 & 0.6 & 1.23998 & 1.23951 \\
\hline 0.6 & 1.23997 & 1.23949 & 0.6 & 1.23999 & 1.23984 & 0.7 & 1.20998 & 1.20962 \\
\hline 0.7 & 1.20998 & 1.20958 & 0.7 & 1.20999 & 1.20986 & 0.8 & 1.15999 & 1.15974 \\
\hline 0.8 & 1.15999 & 1.1597 & 0.8 & 1.15999 & 1.1599 & 0.8 & 1.15999 & 1.15974 \\
\hline 0.9 & 1.08999 & 1.08985 & 0.9 & 1.09 & 1.08995 & 0.9 & 1.08999 & 1.08987 \\
\hline 1 & 1 & 1 & 1 & 1 & 1 & 1 & 1 & 1 \\
\hline
\end{tabular}

Figure 4 shows the effect of wall temperature ratio $m$ on the main velocity $(m=-1$ means that the average of the temperatures of the two walls is equal to that of the static temperature, $m=0$ corresponds to the temperature of the flat wall is equal to static temperature and $m=1$ means that the wavy and flat wall are maintained at equal temperature and $m>1$ implies wall temperatures are unequal). As $m$ increases, main velocity $u$ increases in both streams at the baffle positions $0.2,0.5$ and 0.8 and the flow reversal is observed in stream-2 for $m=-1$. The effect of $m$ on the main velocity is similar to the result obtained by Malashetty et. 
al. (2001) for single passage with wavy wall. Physically, an increase in $m$, increases the temperature and hence buoyancy force increases which results in the increase of velocity field. It is also observed that main velocity profiles for $m=0$ lies in between $m=-1$ (below) and 1 (above). When the baffle position is near left wall, the variations of $m$ are not very effective on main velocity but its effect is dominant when the baffle position moves to the centre and near to the right wall as seen in Figure 4.

The effect of $m$ on the cross velocity is opposite to its effect on main velocity. That is as $m$ increases, cross velocity decreases at all the baffle positions as seen in Figure 5. The velocity profiles for $m=0$ lies in between $m=1$ (below) and -1(above). The effect of $m$ on the cross velocity is not effective for the baffle position at the right wall when compared to the baffle position at the left and at the centre of the channel.

The effect of wall temperature ratio $m$ on the temperature field is shown in Figure 6. As $m$ increases, temperature increases at all the baffle positions and magnitude of promotion also remains the same for $y^{*}=0.2,0.5$ and 0.8 .
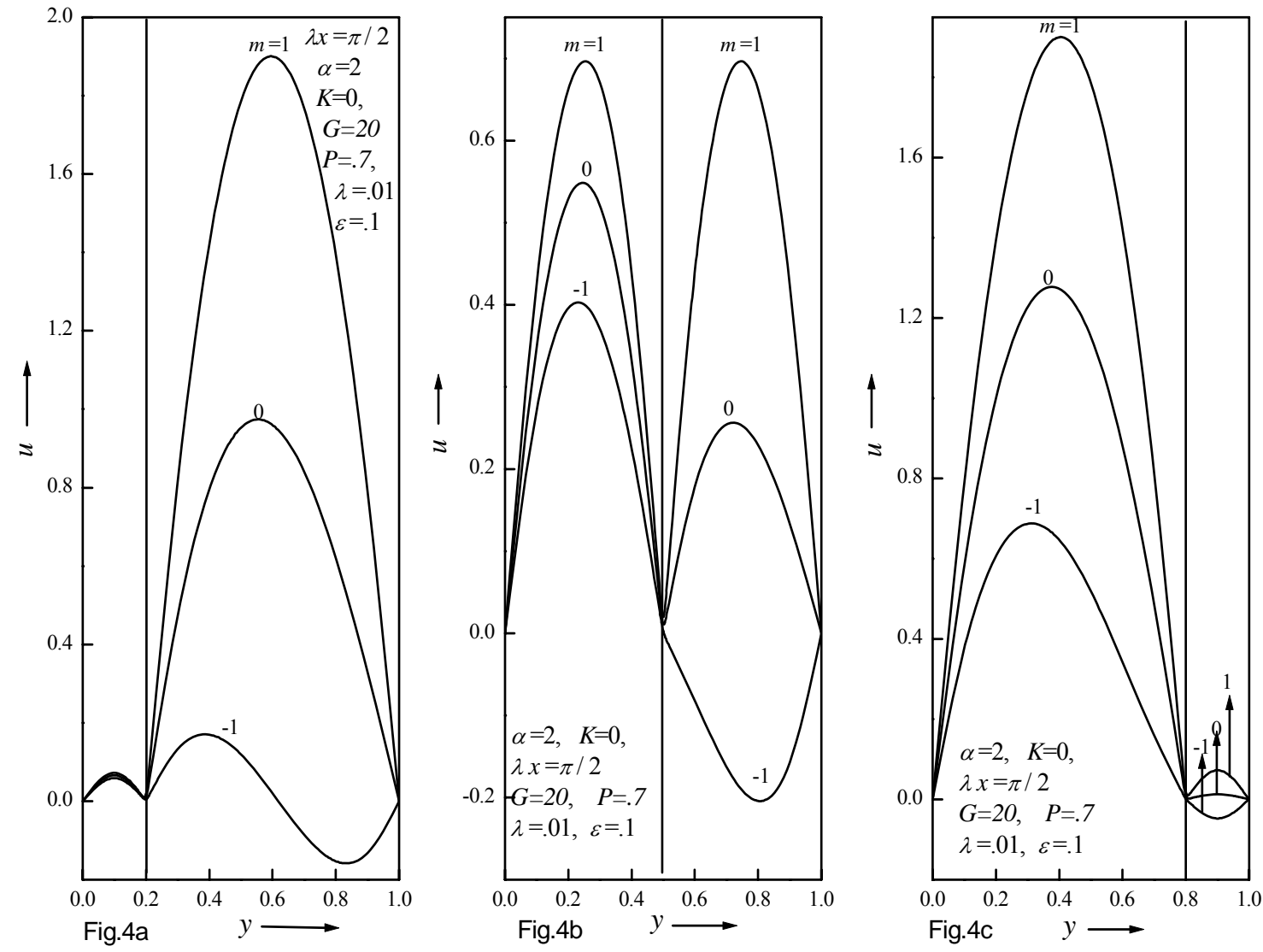

Figure 4: Main velocity profiles for differnt values of wall temperature ratio $m$ 

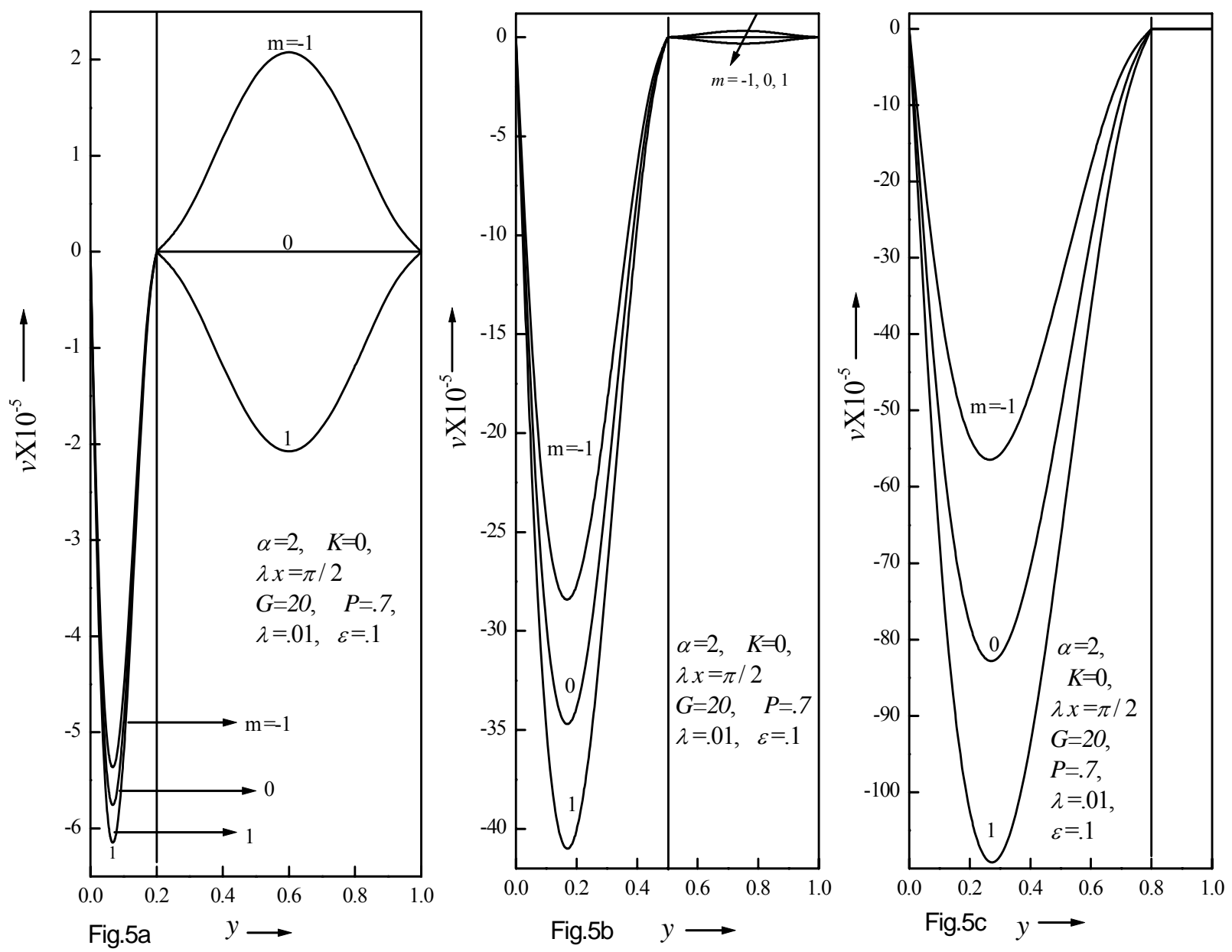

Figure 5: Cross velocity profiles for differnt values of wall temperature ratio $m$. 

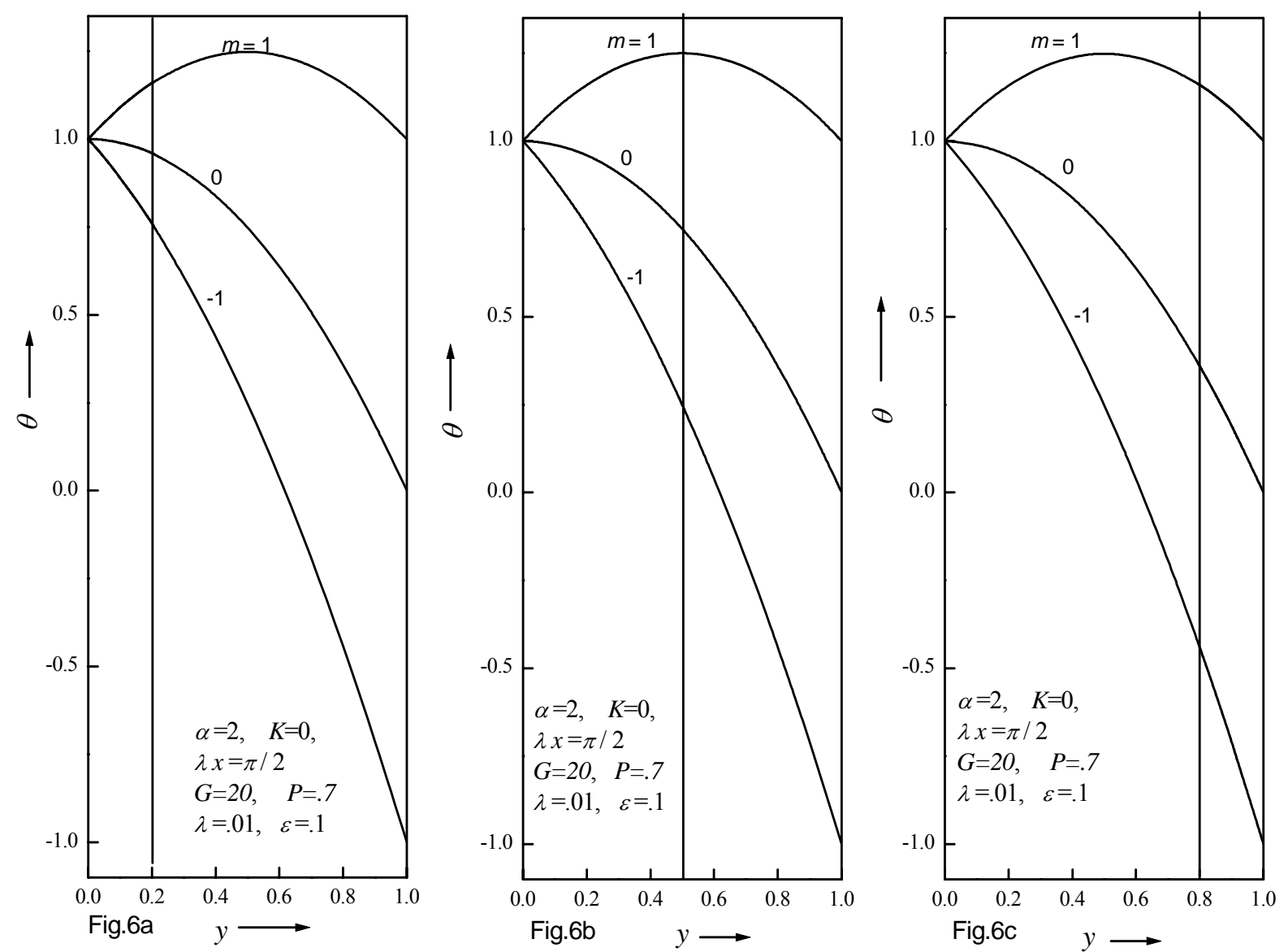

Figure 6: Temperature profiles for differnt values of wall temperature ratio $m$.

The effect of internal heat source $\alpha$ on the main and cross velocity is shown in Figures 7 and 8 respectively. As $\alpha(>0)$ increases the main velocity $u$ increases in both the streams for the baffle positions $y^{*}=0.2,0.5$ and 0.8 . However, the magnitude of promotion is effectively very large in stream-2 when the baffle is placed near the left wall, the magnitude of main velocity is equal when the baffle is in the centre of the channel and the peak value for main velocity is in stream-1 when the baffle is placed near the right wall. The effect of heat sink $\alpha(<0)$ is to reduce the main velocity in both the streams at the baffle positions $y^{*}=0.2,0.5$ and 0.8 as seen in Figure 7. The effect of source $\alpha(>0)$ increases the temperature distribution which enhances the velocity field whereas sink $\alpha(<0)$ suppresses the temperature distribution which causes reduction in the velocity field. As sink parameter increases, reversal flow occurs irrespective of baffle position. The effect of either source or sink on the flow field is similar to the results obtained by Umavathi et. al. (2006) for single passage.

The effect of heat source/sink on cross velocity $v$ is shown in Figure 8 . The effect of heat source/sink on cross velocity is exactly opposite to its effect on main velocity i.e. as $\alpha(>0)$ increases cross velocity decreases and as $\alpha(<0)$ increases cross velocity increases in both the streams. The peak values of cross velocity is in stream- 2 for $y^{*}=0.2,0.5$ and in stream- 1 for $y^{*}=0.8$ as seen in Figure 8.

The effect of heat source $(\alpha>0)$ and heat sink $(\alpha<0)$ on temperature is shown in Figure 9 . As $\alpha(>0)$ increases temperature increases and as $\alpha(<0)$ increases temperature decreases in both the streams. It is also observed that effect of heat source/sink on temperature do not vary with the baffle position.

The effect of viscoelastic parameter $K$ on main and cross velocity is shown in Table $2 \mathrm{a}$ and $2 \mathrm{~b}$ respectively. As the viscoelastic parameter $K$ increases main velocity remains constant up to the order of $10^{-4}$. However there is no effect of viscoelastic parameter $K$ on cross velocity (Table 2b) and on temperature (Table 2c). As $K$ increases main velocity decreases very slightly, 
which is the similar result observed by Rita and Alok (2000) for single passage. That is as $K$ increases skin friction decreases to the order of $10^{-4}$ at both the walls in Rita and Alok (2000).
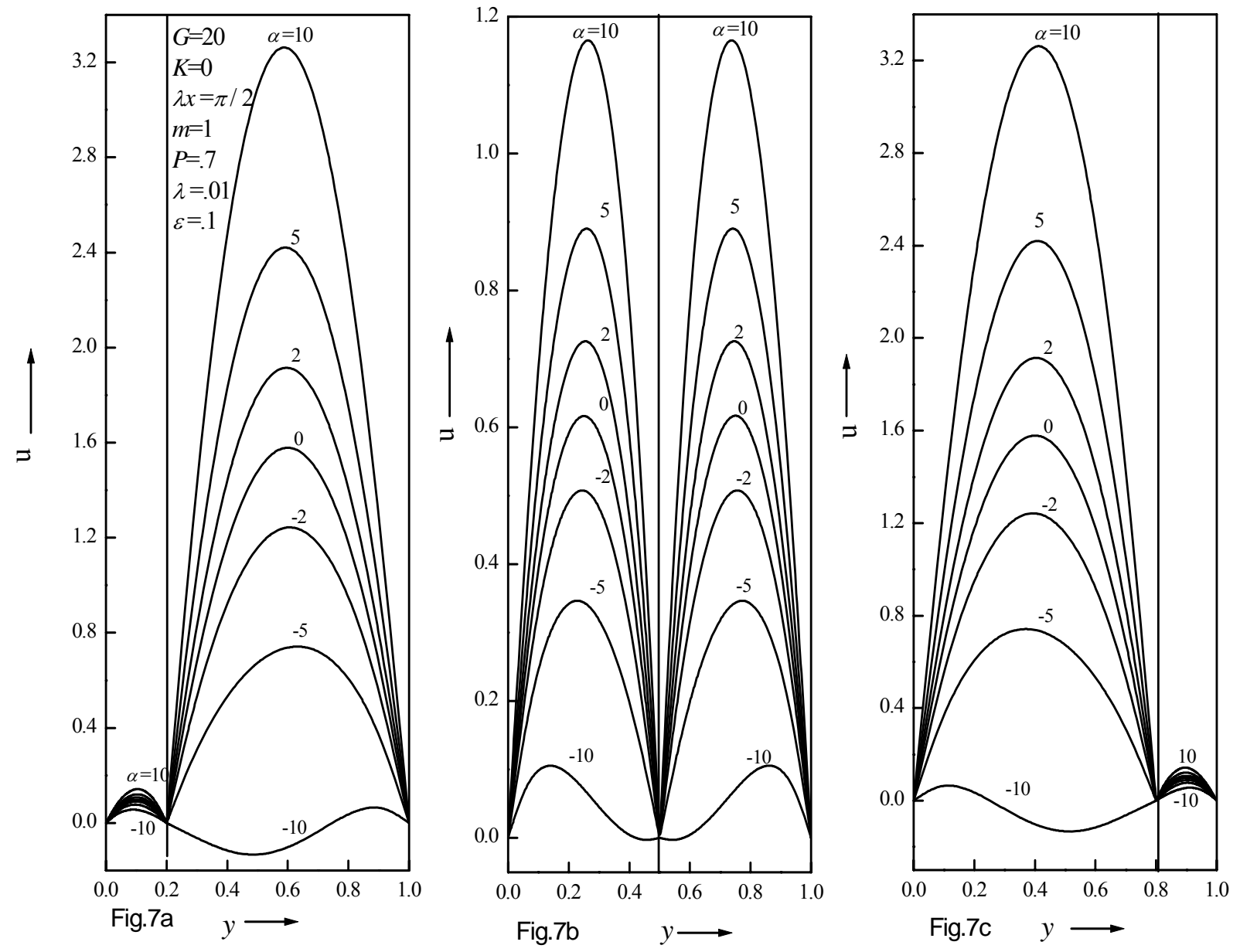

Figure 7: Main velocity profiles for differnt values of heat source/sink $\alpha$. 

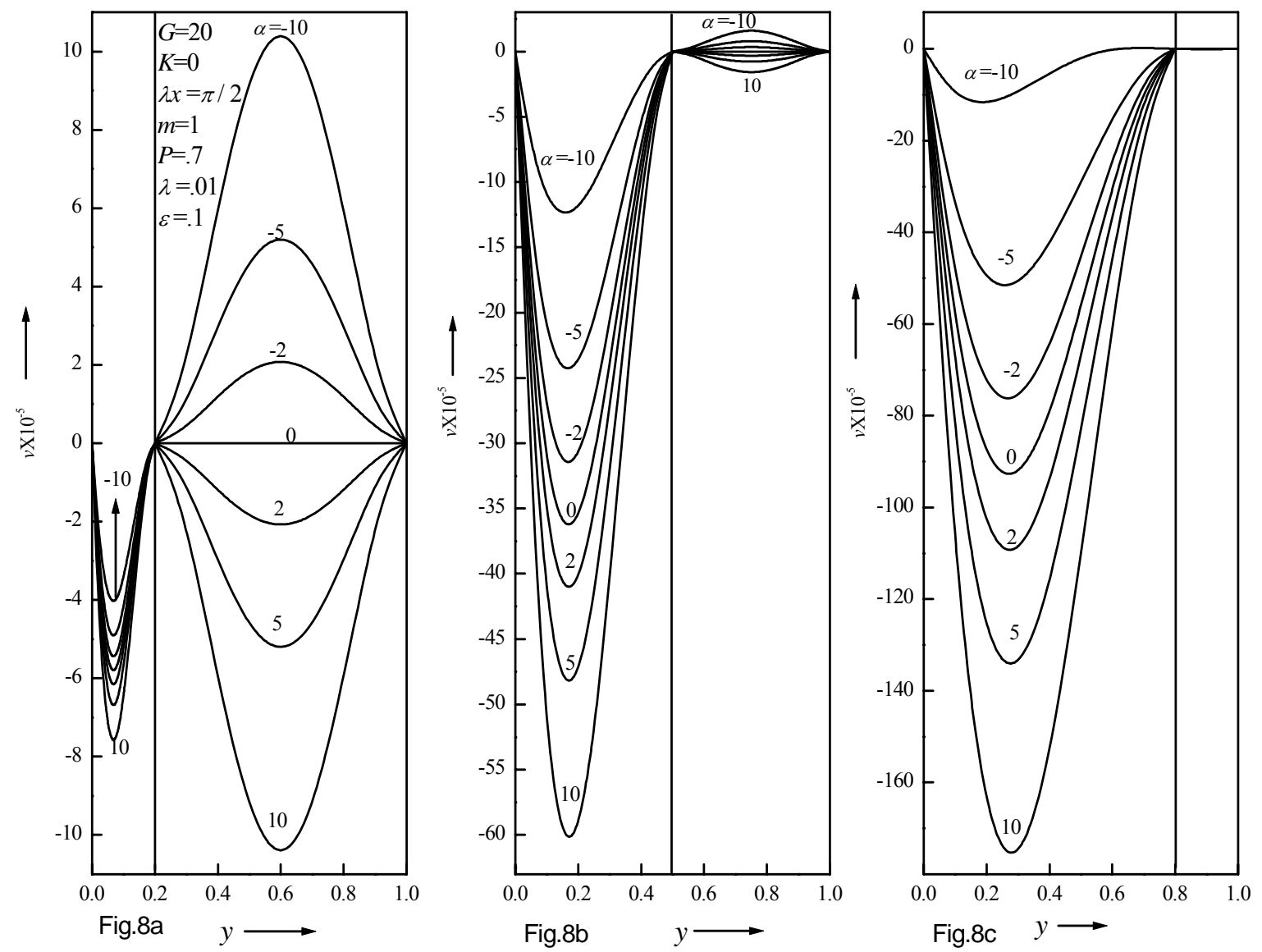

Figure 8: Cross velocity profiles for differnt values of source/sink $\alpha$. 

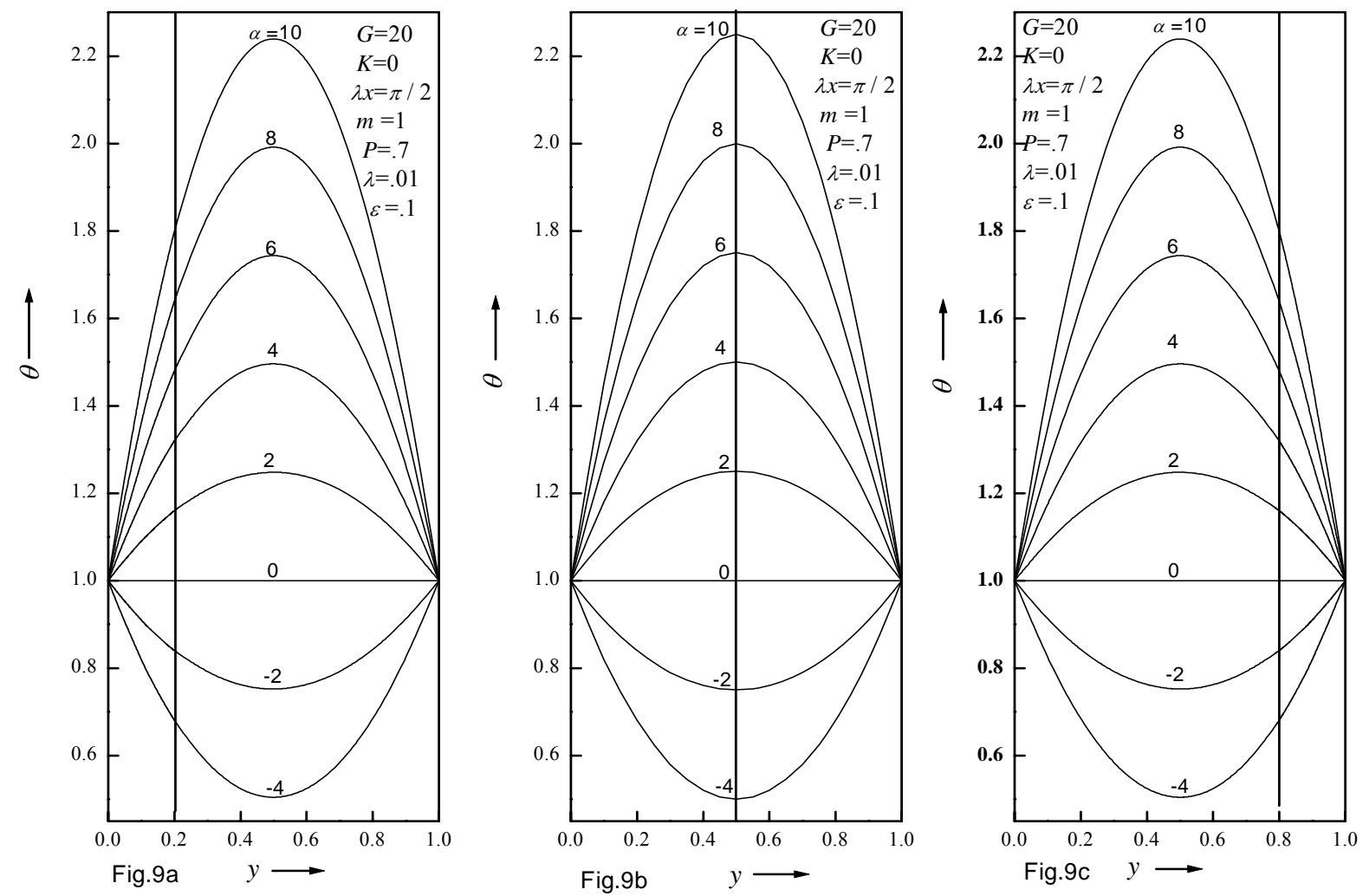

Figure 9: Temperature profiles for differnt values of heat source/sink $\alpha$.

Table 2a. Main velocity for different viscoelastic parameter and baffle position.

\begin{tabular}{|c|c|c|c|}
\hline \multirow{2}{*}{$y$} & \multicolumn{3}{|c|}{$y^{*}=0.2$} \\
\cline { 2 - 4 } 0 & $K=0.0$ & $K=0.15$ & $K=0.25$ \\
\hline 0 & 0 & 0 & 0 \\
\hline 0.1 & 0.1088331235177 & 0.1088331254581 & 0.1088331267518 \\
\hline 0.2 & 0 & 0 & 0 \\
\hline 0.2 & 0 & 0 & 0 \\
\hline 0.3 & 0.8528314350671 & 0.8528482924837 & 0.8528595307615 \\
\hline 0.4 & 1.4639960277544 & 1.4640159751829 & 1.4640292734686 \\
\hline 0.5 & 1.8274958323083 & 1.8275085293440 & 1.8275169940345 \\
\hline 0.6 & 1.9413313793788 & 1.9413313306169 & 1.9413312981090 \\
\hline 0.7 & 1.8075016873718 & 1.8074889304075 & 1.8074804257647 \\
\hline 0.8 & 1.4320047651680 & 1.4319848405966 & 1.4319715575490 \\
\hline 0.9 & 0.8248382713951 & 0.8248214868118 & 0.8248102970896 \\
\hline 1 & 0 & 0 & 0 \\
\hline$y$ & & $y^{*}=0.5$ & \\
\cline { 2 - 4 } & $K=0.0$ & $K=0.15$ & $K=0.25$ \\
\hline 0 & 0 & 0 & 0 \\
\hline 0.1 & 0.4592416468904 & 0.4592416057237 & 0.4592415782793 \\
\hline 0.2 & 0.7009327122020 & 0.7009328992020 & 0.7009330238687 \\
\hline 0.3 & 0.7110328796923 & 0.7110329966923 & 0.7110330746923 \\
\hline 0.4 & 0.4794478480932 & 0.4794476719265 & 0.4794475544821 \\
\hline 0.5 & 0 & 0 & 0 \\
\hline 0.5 & 0 & 0 & 0 \\
\hline 0.6 & 0.4793314574786 & 0.4793333513595 & 0.4793346139468 \\
\hline & & & \\
& & & \\
& & &
\end{tabular}




\begin{tabular}{|c|c|c|c|}
\hline 0.7 & 0.7109987322420 & 0.7109997068134 & 0.7110003565277 \\
\hline 0.8 & 0.7010007559614 & 0.7009997705329 & 0.7009991135805 \\
\hline 0.9 & 0.4593355490563 & 0.4593336629373 & 0.4593324055246 \\
\hline 1 & 0 & 0 & 0 \\
\hline \multirow{2}{*}{$y$} & \multicolumn{3}{|c|}{$y^{*}=0.8$} \\
\cline { 2 - 4 } & $K=0.0$ & $K=0.15$ & $K=0.25$ \\
\hline 0 & 0 & 0 & 0 \\
\hline 0.1 & 0.8240736400061 & 0.8240723974644 & 0.8240715691033 \\
\hline 0.2 & 1.4309904807698 & 1.4309904201984 & 1.4309903798174 \\
\hline 0.3 & 1.8067008853415 & 1.8067025047522 & 1.8067035843593 \\
\hline 0.4 & 1.9410981127098 & 1.9411002826145 & 1.9411017292177 \\
\hline 0.5 & 1.8279949361907 & 1.8279959836014 & 1.8279966818752 \\
\hline 0.6 & 1.4650962110966 & 1.4650952545252 & 1.4650946168109 \\
\hline 0.7 & 0.8539675520714 & 0.8539655535298 & 0.8539642211687 \\
\hline 0.8 & 0 & 0 & 0 \\
\hline 0.8 & 0 & 0 & 0 \\
\hline 0.9 & 0.1088333049525 & 0.1088333049049 & 0.1088333048732 \\
\hline 1 & 0 & 0 & 0 \\
\hline
\end{tabular}

Table 2b. Cross velocity for different viscoelastic parameter and baffle position.

\begin{tabular}{|c|c|c|c|c|c|}
\hline \multirow{2}{*}{$y$} & $y^{*}=0.2$ & \multirow{2}{*}{$y$} & $y^{*}=0.5$ & \multirow{2}{*}{$y$} & $y^{*}=0.8$ \\
\cline { 6 - 6 } & $K=0.0,0.15,0.25$ & & $K=0.0,0.15,0.25$ & & $K=0.0,0.15,0.25$ \\
\hline 0 & 0 & 0 & 0 & 0 & $-7.145833 \times 10^{-4}$ \\
\hline 0.1 & $-5.308333 \times 10^{-5}$ & 0.1 & $-3.613333 \times 10^{-4}$ & 0.1 & -0.0010560 \\
\hline 0.2 & 0 & 0.2 & $-4.08 \mathrm{E}-4$ & 0.2 & -0.00110625 \\
\hline 0.2 & 0 & 0.3 & $-2.73 \mathrm{E}-4$ & 0.3 & $-9.49333 \times 10^{-4}$ \\
\hline 0.3 & $-4.083337 \mathrm{X} 10^{-6}$ & 0.4 & $-9.133333 \times 10^{-5}$ & 0.4 & $-6.7125 \times 10^{-4}$ \\
\hline 0.4 & $-1.2 \mathrm{E}-5$ & 0.5 & 0 & 0.5 & $-3.6 \mathrm{E}-4$ \\
\hline 0.5 & $-1.875 \mathrm{E}-5$ & 0.5 & 0 & 0.6 & $-1.055833 \times 10^{-4}$ \\
\hline 0.6 & $-2.133334 \mathrm{X} 10^{-5}$ & 0.6 & $-1.3333 \times 10^{-6}$ & 0.7 & 0 \\
\hline 0.7 & $-1.875 \mathrm{E}-5$ & 0.7 & $-2.99998 \times 10^{-6}$ & 0.8 & 0 \\
\hline 0.8 & $-1.2 \mathrm{E}-5$ & 0.8 & $-3.00001 \times 10^{-6}$ & 0.8 & $-8.33546 \times 10^{-8}$ \\
\hline 0.9 & $-4.08331 \times 10^{-6}$ & 0.9 & $-1.33331 \times 10^{-6}$ & 0.9 & $1.1102 \times 10^{-19}$ \\
\hline 1 & 0 & 1 & 0 & 1 & $-7.14583 \times 10^{-4}$ \\
\hline
\end{tabular}

Table 2c. Temperature values for different viscoelastic parameter and baffle position.

\begin{tabular}{|c|c|c|c|c|c|}
\hline \multirow{2}{*}{$y$} & $y^{*}=0.2$ & \multirow{2}{*}{$y$} & $y^{*}=0.5$ & \multirow{2}{*}{$y$} & $y^{*}=0.8$ \\
\cline { 2 - 2 } \cline { 6 - 6 } & $K=0.0,0.15,0.25$ & & $K=0.0,0.15,0.25$ & & $K=0.0,0.15,0.25$ \\
\hline 0 & 1.000000000 & 0 & 1.000000000 & 0 & 1.000000000 \\
\hline 0.1 & 1.089985643 & 0.1 & 1.089993097 & 0.1 & 1.089982338 \\
\hline 0.2 & 1.159971603 & 0.2 & 1.159987100 & 0.2 & 1.159965967 \\
\hline 0.2 & 1.159971603 & 0.3 & 1.209983242 & 0.3 & 1.209953161 \\
\hline 0.3 & 1.209958492 & 0.4 & 1.239981989 & 0.4 & 1.239946019 \\
\hline 0.4 & 1.239949365 & 0.5 & 1.249982530 & 0.5 & 1.249945576 \\
\hline 0.5 & 1.249946228 & 0.5 & 1.249982530 & 0.6 & 1.239951412 \\
\hline 0.6 & 1.239949388 & 0.6 & 1.239983697 & 0.7 & 1.209961790 \\
\hline 0.7 & 1.209957956 & 0.7 & 1.209986108 & 0.8 & 1.159974288 \\
\hline 0.8 & 1.159970358 & 0.8 & 1.159989966 & 0.8 & 1.159974288 \\
\hline 0.9 & 1.089984843 & 0.9 & 1.089994804 & 0.9 & 1.089987112 \\
\hline 1 & 1.000000000 & 1 & 1.000000000 & 1 & 1.000000000 \\
\hline
\end{tabular}

The effect of $\lambda x$ on main velocity is shown in Figure 10. As $\lambda x$ increases, main velocity increases near the wavy wall and reverses its direction near the baffle position and remains constant at the flat wall at all baffle positions

The effect of $\lambda x$ on cross velocity is shown in Figure 11. As $\lambda x$ increases, cross velocity decreases in stream 1 and stream-2 for the baffle positions at the left, centre and right walls. However there is no effect of $\lambda x$ at the right wall when $y^{*}=0.5,0.8$. 
The effect of $\lambda x$ on the temperature field is shown in Figure 12. As $\lambda x$ increases, temperature increases at all the baffle positions and magnitude of promotion also remains the same for $y^{*}=0.2,0.5$ and 0.8 . The effect of the product of nondimensional wave length and space co-ordinate on the temperature is the similar result obtained by Jang et. al. (2003) and Wang and Vanka (1995) for single passage. Jang et al. (2003) showed that, as the wave length increases, rate of heat transfer increases at the wavy wall. Wang and Vanka (1995) observed that in the steady flow regime, the average Nusselt number for the wavy wall channel were larger than those for a parallel plate channel.
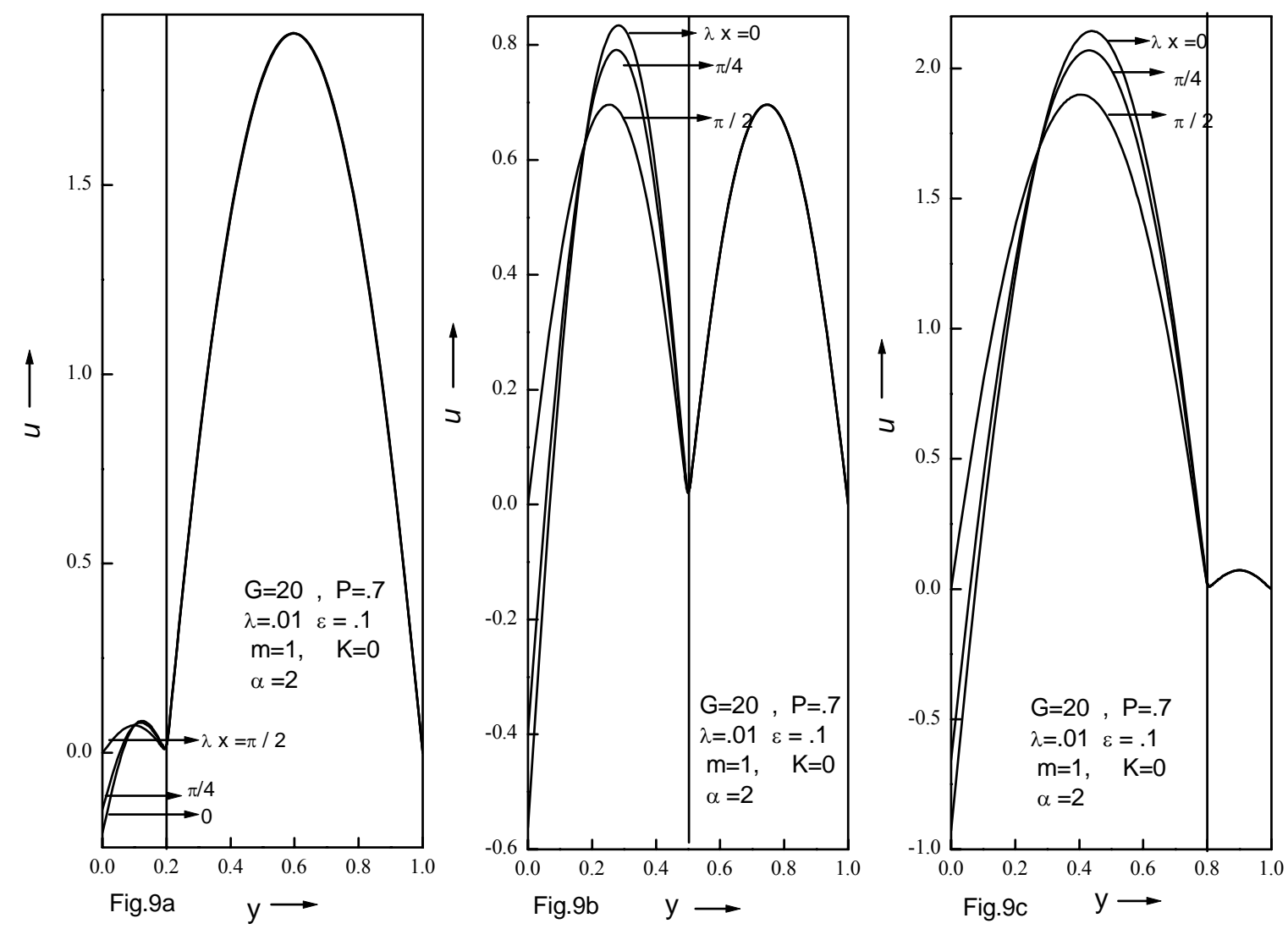

Figure 10: Main velocity profiles for different values of $\lambda x$. 

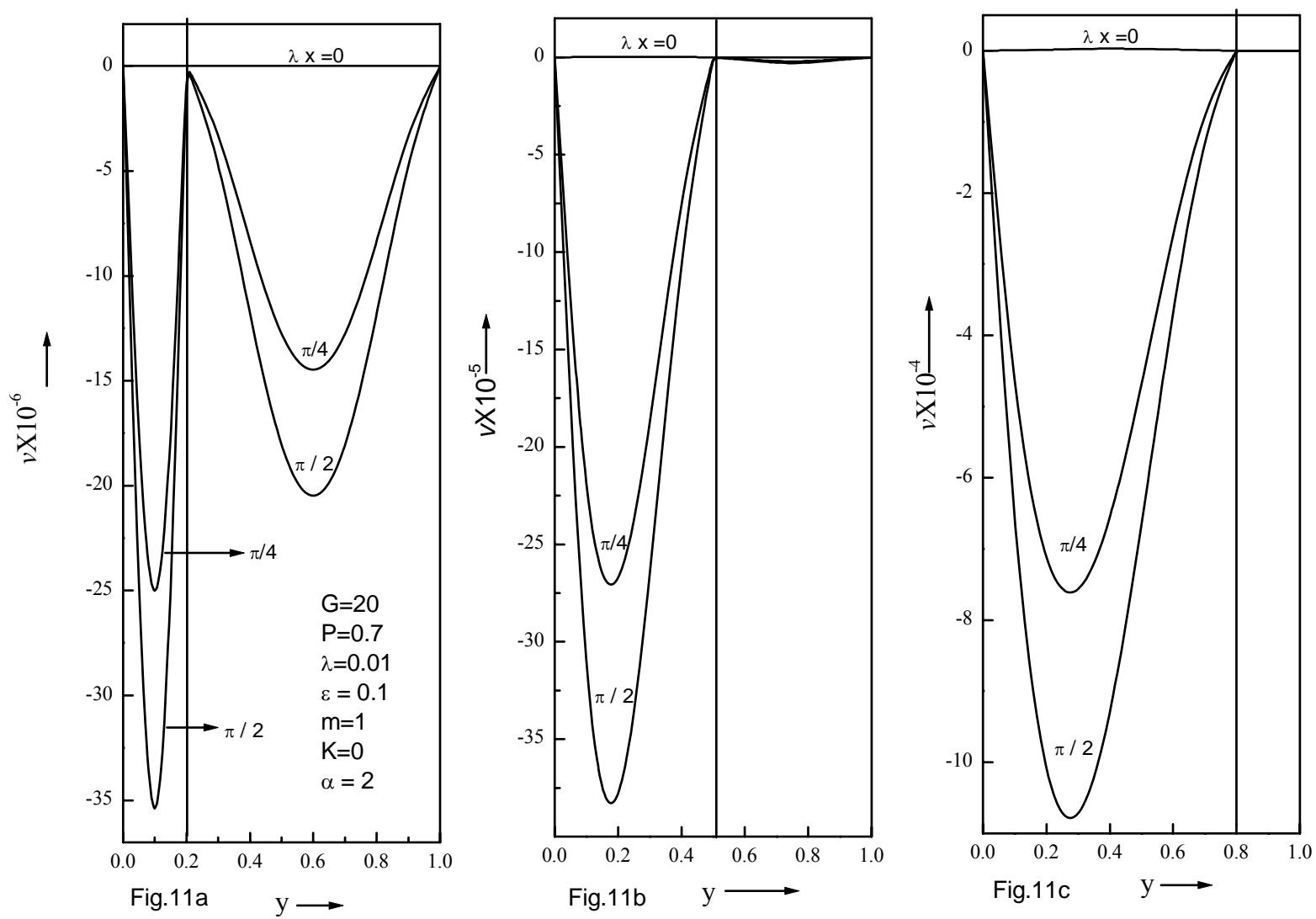

Figure 11: Cross velocity profiles for different values of $\lambda x$.

The values of skin friction and Nusselt numbers on different physical parameters are shown in Tables $3 \mathrm{a}$ and $3 \mathrm{~b}$ respectively for the values of $G=20, K=0.0, m=1, \lambda=0.01, \varepsilon=0.1$ except the varying parameter. The skin friction increases at the wavy wall and decreases at the flat wall for increasing Grashof number, heat source/sink and wall temperature ratio for the wider passage. The viscoelastic parameter, wave number and amplitude parameter reduces the skin friction at both the walls.

The effect of Grashof number, wave number and the amplitude parameter is to decrease the Nusselt number at the wavy wall and increase at the flat wall, while the effect of heat source/sink enhances the Nusselt number at the wavy wall and reduces at the flat wall. The effect of viscoelastic parameter remains constant at both the walls at all baffle positions. The Nusselt number increases at both the walls with increase of wall temperature ratio. 

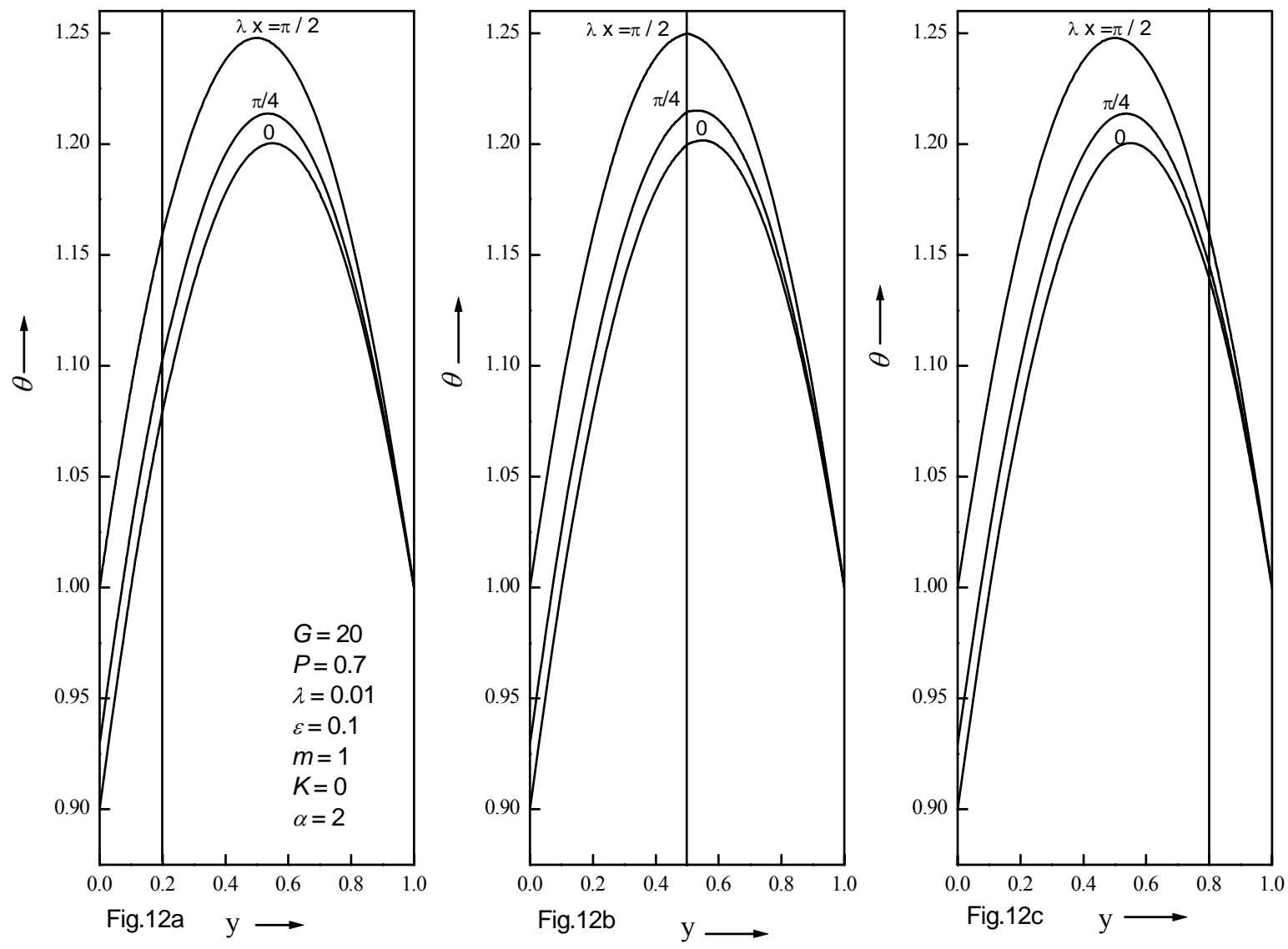

Figure 12: Temperature profiles for different values of $\lambda x$.

Table 3a. skin friction values at the wavy and flat wall.

\begin{tabular}{|c|c|c|l|l|l|l|l|}
\hline & \multicolumn{3}{|c|}{$\sigma_{w}$} & \multicolumn{3}{c|}{$\sigma_{f}$} \\
\hline$G$ & $y^{*}=0.2$ & $y^{*}=0.5$ & $y^{*}=0.8$ & $G$ & $y^{*}=0.2$ & $y^{*}=0.5$ & $y^{*}=0.8$ \\
\hline 20 & 2.12001 & 5.62498 & 9.2798 & 20 & -9.28008 & -5.62505 & -2.12003 \\
\hline 100 & 10.6002 & 28.1245 & 46.395 & 100 & -46.402 & -28.1264 & -10.6008 \\
\hline 200 & 21.2009 & 56.2479 & 92.7801 & 200 & -92.8082 & -56.2555 & -21.2032 \\
\hline$m$ & & & & $m$ & & & \\
\hline-1 & 1.85333 & 3.95832 & 5.01335 & -1 & 2.45367 & 2.70859 & 1.61342 \\
\hline 0 & 1.98667 & 4.79165 & 7.14659 & 0 & -3.41333 & -1.45834 & -0.253338 \\
\hline 1 & 2.12001 & 5.62948 & 9.2798 & 1 & -9.28008 & -5.62505 & -2.12003 \\
\hline$K$ & & & & $K$ & & & \\
\hline 0 & 2.12001 & 5.62948 & 9.2798 & 0 & -9.28008 & -5.62505 & -2.12003 \\
\hline 0.15 & 2.11866 & 5.61548 & 9.25394 & 0.15 & -9.27984 & -5.62502 & -2.12003 \\
\hline 0.25 & 2.11776 & 5.60915 & 9.23669 & 0.25 & -9.27968 & -5.62499 & -2.12003 \\
\hline$\lambda$ & & & & $\lambda$ & & & \\
\hline 0 & 2.12 & 5.625 & 9.28 & 0 & -9.28 & -5.625 & -2.12 \\
\hline 0.1 & 2.12009 & 5.62479 & 9.27801 & 0.1 & -9.28082 & -5.62555 & -2.12032 \\
\hline 0.5 & 2.12043 & 5.62393 & 9.27006 & 0.5 & -9.28409 & -5.62773 & -2.12158 \\
\hline$\varepsilon$ & & & & $\varepsilon$ & & & \\
\hline 0 & 2.12 & 5.625 & 9.28 & 0 & -9.28 & -5.625 & -2.12 \\
\hline 0.1 & 2.12001 & 5.62498 & 9.2798 & 0.1 & -9.28008 & -5.62505 & -2.12003 \\
\hline 0.5 & 2.12004 & 5.62489 & 9.27901 & 0.5 & -9.28041 & -5.62527 & -2.12016 \\
\hline
\end{tabular}


Table 3b. Nusselt number values at the wavy and flat wall.

\begin{tabular}{|c|l|l|l|l|l|l|l|}
\hline & \multicolumn{5}{|c}{$N u_{w}$} & & \multicolumn{3}{c|}{$N u_{f}$} \\
\hline$G$ & \multicolumn{1}{|c|}{$y^{*}=0.2$} & $y^{*}=0.5$ & $y^{*}=0.8$ & $G$ & $y^{*}=0.2$ & $y^{*}=0.5$ & $y^{*}=0.8$ \\
\hline 20 & 0.999856 & 0.99993 & 0.999822 & 20 & -0.99984 & -0.99994 & -0.99987 \\
\hline 100 & 0.99928 & 0.999651 & 0.999111 & 100 & -0.99924 & -0.99973 & -0.99935 \\
\hline 200 & 0.998559 & 0.999301 & 0.998223 & 200 & -0.99847 & -0.999477 & -0.99871 \\
\hline$m$ & & & & $m$ & & & \\
\hline-1 & -0.99998 & -1.00001 & -1.00008 & -1 & -3.0001 & -2.99999 & -2.99995 \\
\hline 0 & $-6.67 X 10^{-7}$ & $-2.09 X 10^{-5}$ & $-1.024 \mathrm{E}-4$ & 0 & -2.000 & -1.99999 & -1.99993 \\
\hline 1 & 0.999856 & 0.99993 & 0.999822 & 1 & -0.99984 & -0.99994 & -0.99987 \\
\hline$K$ & & & & $K$ & & & \\
\hline 0 & 0.999856 & 0.99993 & 0.999822 & 0 & -0.99984 & -0.99994 & -0.99987 \\
\hline 0.15 & 0.999856 & 0.99993 & 0.999822 & 0.15 & -0.99984 & -0.99994 & -0.99987 \\
\hline 0.25 & 0.999856 & 0.99993 & 0.999822 & 0.25 & -0.99984 & -0.99994 & -0.99987 \\
\hline$\lambda$ & & & & $\lambda$ & & & \\
\hline 0 & 1.0 & 1.0 & 1.0 & 0 & -1.0 & -1.0 & -1.0 \\
\hline 0.1 & 0.998559 & 0.999301 & 0.998223 & 0.1 & -0.99847 & -0.99947 & -0.99871 \\
\hline 0.5 & 0.992797 & 0.996506 & 0.991113 & 0.5 & -0.99239 & -0.99738 & -0.99355 \\
\hline$\varepsilon$ & & & & $\varepsilon$ & & & \\
\hline 0 & 1.0 & 1.0 & 1.0 & 0 & -1.0 & -1.0 & -1.0 \\
\hline 0.1 & 0.999856 & 0.99993 & 0.999822 & 0.1 & -0.99984 & -0.99994 & -0.99987 \\
\hline 0.5 & 0.99928 & 0.99965 & 0.999111 & 0.5 & -0.99924 & -0.99973 & -0.99935 \\
\hline
\end{tabular}

The results obtained in case 2a and the results obtained by Salah El Din (2002) are displayed in Table 4. There is a very good agreement for the values of velocity at different baffle positions which will justify the present model with baffle.

Table 4. Comparison of velocity at different baffle positions with Salah El-Din (2002) for

\begin{tabular}{|c|c|c|c|c|c|c|c|c|}
\hline \multirow[t]{2}{*}{$y$} & $\begin{array}{c}\text { Present } \\
\text { Model }\end{array}$ & $\begin{array}{l}\text { Salah } \\
\text { El-Din } \\
(2002)\end{array}$ & \multirow[t]{2}{*}{$y$} & $\begin{array}{l}\text { Present } \\
\text { Model }\end{array}$ & $\begin{array}{l}\text { Salah } \\
\text { El-Din } \\
(2002)\end{array}$ & \multirow[t]{2}{*}{$y$} & $\begin{array}{l}\text { Present } \\
\text { Model }\end{array}$ & $\begin{array}{c}\text { Salah } \\
\text { El-Din } \\
(2002)\end{array}$ \\
\hline & $y^{*}=0.2$ & $y^{*}=0.2$ & & $y^{*}=0.5$ & $y^{*}=0.5$ & & $y^{*}=0.8$ & $y^{*}=0.8$ \\
\hline 0 & 0 & 0 & 0 & 0 & 0 & 0.1 & 0 & 0 \\
\hline 0.1 & -0.02 & -0.02 & 0.1 & -0.06 & -0.06 & 0.2 & -0.07 & -0.07 \\
\hline 0.2 & 0 & 0 & 0.2 & -0.08 & -0.08 & 0.3 & -0.1 & -0.1 \\
\hline 0.2 & 0 & 0 & 0.3 & -0.07 & -0.07 & 0.4 & -0.1 & -0.1 \\
\hline 0.3 & 0 & 0 & 0.4 & -0.04 & -0.04 & 0.5 & -0.08 & -0.08 \\
\hline 0.4 & 0.02 & 0.02 & 0.5 & 0 & 0 & 0.6 & -0.05 & -0.05 \\
\hline 0.5 & 0.05 & 0.05 & 0.5 & 0 & 0 & 0.7 & -0.02 & -0.02 \\
\hline 0.6 & 0.08 & 0.08 & 0.6 & 0.04 & 0.04 & 0.8 & 0 & 0 \\
\hline 0.7 & 0.1 & 0.1 & 0.7 & 0.07 & 0.07 & 0.8 & 0 & 0 \\
\hline 0.8 & 0.1 & 0.1 & 0.8 & 0.08 & 0.08 & 0.9 & 0 & 0 \\
\hline 0.9 & 0.07 & 0.07 & 0.9 & 0.06 & 0.06 & 1 & 0.02 & 0.02 \\
\hline 1 & 0 & 0 & 1 & 0 & 0 & 0 & 0 & 0 \\
\hline
\end{tabular}

The solutions obtained in case $2 \mathrm{~b}$ and the solutions obtained by Rita and Alok (2000) are shown in Table 5. When the baffle is shifted to the right wall (stream-1), the problem reduced to single passage whose solutions agree with Rita and Alok (2000) which justifies the solutions of stream- 1 . To justify the solutions obtained in stream-2, the baffle is shifted to the right wall, which again reduces to single passage. The solutions obtained in stream-2 agree very well with Rita and Alok (2000). 
Table 5. Validity of the present model with stream-1 (shifting the baffle to the right wall) and stream-2 (shifting the baffle to the left wall) with Rita and Alok (2000) for $G=10, m=1, K=0, \alpha=2, P=0.7 \quad$ and $\lambda=0.01$.

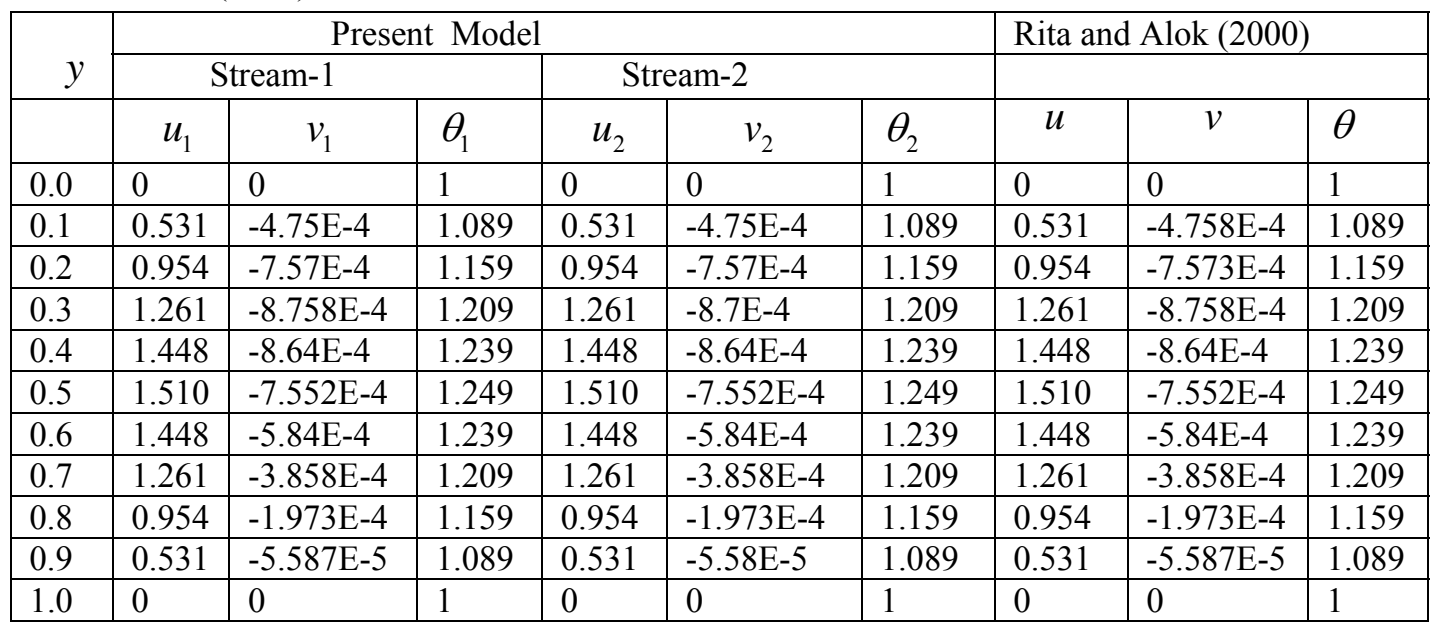

\section{Conclusion}

The characteristics of flow and heat transfer of viscoelastic (Walters Fluid Model B') fluid in a vertical channel one of whose walls is wavy in a double-passage channel with a perfectly conducting baffle are investigated. According to the results the following conclusions can be drawn.

1. The maxima of main velocity profiles are obtained for increasing values of Grashof number, wall temperature ratio, heat source/sink especially in the wider passage. The effect of viscoelastic parameter reduces the main velocity in stream- 1 and increases stream-2 for wider passage with equal wall temperature. As $\lambda x$ increases, the main velocity increases at the wavy wall and remains constant at the flat wall at all baffle positions. The effects of Grashof number, wall temperature ratio and $\lambda x$ on cross velocity are exactly opposite to their effect on main velocity. The effect of viscoelastic parameter on cross velocity remains invariant.

2. The temperature profiles remain almost invariant with changes in the Grashof number and viscoelastic parameter at all baffle positions. The effect of wall temperature ratio promotes the temperature and $\lambda x$ also increases the temperature at the wavy wall and remains constant at the flat wall.

3. The skin friction increases at the wavy wall and decreases at the flat wall for increasing Grashof number, heat source/sink and wall temperature ratio for the wider passage. The viscoelastic parameter, wave number and amplitude parameter reduces the skin friction at both the walls.

4. The effect of Grashof number, wave number and the amplitude parameter is to decrease the Nusselt number at the wavy wall and increase at the flat wall, while the effect of heat source/sink enhances the Nusselt number at the wavy wall and reduces at the flat wall. The effect of viscoelastic parameter remains constant at both the walls at all baffle positions. The Nusselt number increases at both the walls with increase of wall temperature ratio.

5. The results of the present model agreed very well with the results obtained by Salah El Din (2002) for flat wall and for viscous fluid in the presence of baffle.

6. The results of the present model were in good agreement with the results of Rita and Alok (2000) for single passage with wavy wall for Walter's fluid B'model.

\section{Nomenclature}

$d \quad$ channel width $(\mathrm{m})$

$d^{*} \quad$ width of passage $1(\mathrm{~m})$

$C_{p} \quad$ dimensionless specific heat at constant pressure

$g_{x} \quad$ acceleration due to gravity $\left(\mathrm{ms}^{-2}\right)$

G Grashof number $\left(d^{3} g_{x} \beta\left(T_{w}-T_{s}\right) / v^{2}\right)$

$K \quad$ dimensionless viscoelastic parameter

$k \quad$ wavelength $(\mathrm{m})$

$m \quad$ wall temperature ratio

$\mathrm{Nu} \quad$ Nusselt number 


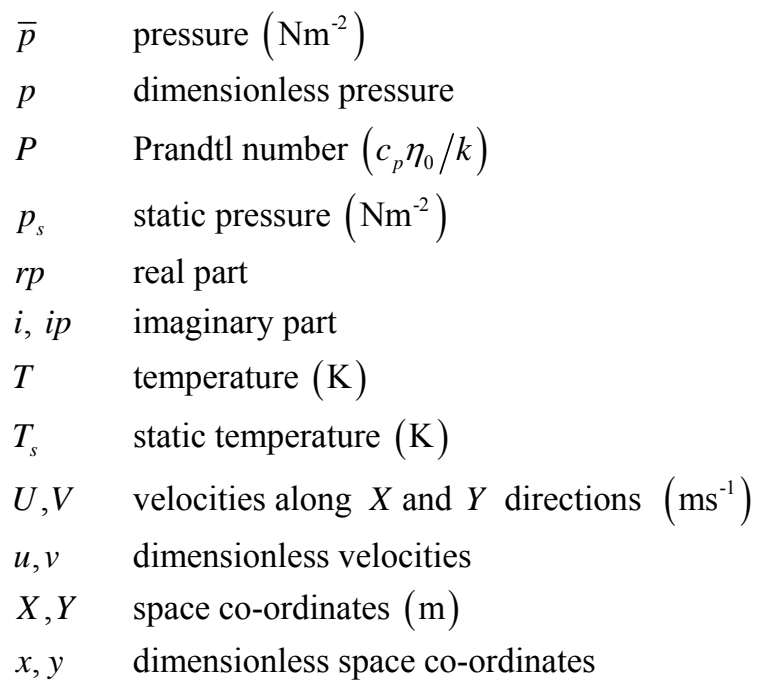

\section{Greek Symbols}

$\beta \quad$ dimensionless co-efficient of thermal expansion

$\varepsilon \quad$ non-dimensional amplitude parameter $\left(\varepsilon^{*} / d\right)$

$\varepsilon^{*} \quad$ amplitude $(\mathrm{m})$

$\lambda \quad$ non-dimensional wave number $(k d)$

$\mu \quad$ viscosity $\left(\mathrm{kg} \mathrm{m}^{-1} \mathrm{~s}^{-1}\right)$

$v \quad$ kinematic viscosity

$\theta$ dimensionless temperature

$\alpha \quad$ the non-dimensional heat source/sink parameter $\left(Q d^{2} / k\left(T_{w}-T_{s}\right)\right)$

$\rho \quad$ density $\left(\mathrm{kg} \mathrm{m}^{-3}\right)$

$\rho_{0} \quad$ static density $\left(\mathrm{kg} \mathrm{m}^{-3}\right)$

$\sigma_{x y} \quad$ skin friction

$\psi \quad$ stream function

\section{Subscripts}

$j$ refers to quantities for the fluids in stream- 1 and stream-2 respectively.

\section{Appendix}

\section{Case 1: Free convection of Walter's fluid in a vertical channel with baffle}

$$
\begin{aligned}
& \theta_{10}=\frac{-\alpha y^{2}}{2}+c_{1} y+c_{2} \\
& u_{10}=l_{1} y^{4}+l_{2} y^{3}+l_{3} y^{2}+d_{1} y+d_{2} \\
& \theta_{20}=\frac{-\alpha y^{2}}{2}+c_{7} y+c_{8} \\
& u_{20}=l_{7} y^{4}+l_{8} y^{3}+l_{9} y^{2}+d_{11} y+d_{12} \\
& u_{11}=-\cos (\lambda x)\left(4 l_{4} y^{3}+\frac{d_{3}}{2} y^{2}+d_{4} y+d_{5}\right)+\lambda \sin (\lambda x)\left(\frac{n_{1}}{504} y^{9}+\frac{n_{2}}{336} y^{8}+\frac{n_{3}}{210} y^{7}+\frac{n_{4}}{120} y^{6}+\frac{n_{5}}{60} y^{5}\right. \\
& \left.+\frac{n_{6}}{24} y^{4}+\frac{n_{7}}{6} y^{3}+\frac{n_{8}}{6} y^{3}+\frac{d_{7}}{2} y^{2}+d_{8} y+d_{9}\right)
\end{aligned}
$$




$$
\begin{aligned}
& u_{21}=-\cos (\lambda x)\left(4 l_{10} y^{3}+\frac{d_{13}}{2} y^{2}+d_{14} y+d_{15}\right)+\lambda \sin (\lambda x)\left(\frac{n_{11}}{504} y^{9}+\frac{n_{12}}{336} y^{8}+\frac{n_{13}}{210} y^{7}+\frac{n_{14}}{120} y^{6}+\frac{n_{15}}{60} y^{5}+\frac{n_{16}}{24} y^{4}\right. \\
& \left.+\frac{n_{17}}{6} y^{3}+\frac{n_{18}}{6} y^{3}+\frac{d_{17}}{2} y^{2}+d_{18} y+d_{19}\right) \\
& v_{11}=-\lambda \sin (\lambda x)\left(l_{4} y^{4}+\frac{d_{3}}{6} y^{3}+\frac{d_{4}}{2} y^{2}+d_{5} y+d_{6}\right)-\lambda^{2} \cos (\lambda x)\left(\frac{n_{1}}{5040} y^{10}+\frac{n_{2}}{3024} y^{9}+\frac{n_{3}}{1680} y^{8}+\right. \\
& \left.\frac{n_{4}}{840} y^{7}+\frac{n_{5}}{360} y^{6}+\frac{n_{6}}{120} y^{5}+\frac{n_{7}}{24} y^{4}+\frac{n_{8}}{24} y^{4}+\frac{d_{7}}{6} y^{3}+\frac{d_{8}}{2} y^{2}+d_{9} y+d_{10}\right) \\
& v_{21}=-\lambda \sin (\lambda x)\left(l_{10} y^{4}+\frac{d_{13}}{6} y^{3}+\frac{d_{14}}{2} y^{2}+d_{15} y+d_{16}\right)-\lambda^{2} \cos (\lambda x)\left(\frac{n_{11}}{5040} y^{9}+\frac{n_{12}}{3024} y^{9}+\frac{n_{13}}{1680} y^{8}+\frac{n_{14}}{840} y^{7}\right. \\
& \left.+\frac{n_{15}}{360} y^{6}+\frac{n_{16}}{120} y^{5}+\frac{n_{17}}{24} y^{4}+\frac{n_{18}}{24} y^{4}+\frac{d_{17}}{6} y^{3}+\frac{d_{18}}{2} y^{2}+d_{19} y+d_{20}\right) \\
& \theta_{11}=\cos (\lambda x)\left(c_{3} y+c_{4}\right)-\lambda \sin (\lambda x)\left(P\left(m_{1} y^{7}+m_{2} y^{6}+m_{3} y^{5}+m_{4} y^{4}+m_{5} y^{3}+m_{6} y^{2}\right)+c_{5} y+c_{6}\right) \\
& \theta_{21}=\cos (\lambda x)\left(c_{9} y+c_{10}\right)-\lambda \sin (\lambda x)\left(P\left(m_{7} y^{7}+m_{8} y^{6}+m_{9} y^{5}+m_{10} y^{4}+m_{11} y^{3}+m_{12} y^{2}\right)+c_{11} y+c_{12}\right) \\
& u_{1}=\left(l_{1} y^{4}+l_{2} y^{3}+l_{3} y^{2}+d_{1} y+d_{2}\right)+\varepsilon\left(-\cos (\lambda x)\left(4 l_{4} y^{3}+\frac{d_{3}}{2} y^{2}+d_{4} y+d_{5}\right)+\lambda \sin (\lambda x)\left(\frac{n_{1}}{504} y^{9}+\frac{n_{2}}{336} y^{8}\right.\right. \\
& \left.\left.+\frac{n_{3}}{210} y^{7}+\frac{n_{4}}{120} y^{6}+\frac{n_{5}}{60} y^{5}+\frac{n_{6}}{24} y^{4}+\frac{n_{7}}{6} y^{3}+\frac{n_{8}}{6} y^{3}+\frac{d_{7}}{2} y^{2}+d_{8} y+d_{9}\right)\right) \\
& u_{2}=\left(l_{7} y^{4}+l_{8} y^{3}+l_{9} y^{2}+d_{11} y+d_{12}\right)+\varepsilon\left(-\cos (\lambda x)\left(4 l_{10} y^{3}+\frac{d_{13}}{2} y^{2}+d_{14} y+d_{15}\right)+\lambda \sin (\lambda x)\left(\frac{n_{11}}{504} y^{9}\right.\right. \\
& \left.\left.+\frac{n_{12}}{336} y^{8}+\frac{n_{13}}{210} y^{7}+\frac{n_{14}}{120} y^{6}+\frac{n_{15}}{60} y^{5}+\frac{n_{16}}{24} y^{4}+\frac{n_{17}}{6} y^{3}+\frac{n_{18}}{6} y^{3}+\frac{d_{17}}{2} y^{2}+d_{18} y+d_{19}\right)\right) \\
& v_{1}=\varepsilon\left(-\lambda \sin (\lambda x)\left(l_{4} y^{4}+\frac{d_{3}}{6} y^{3}+\frac{d_{4}}{2} y^{2}+d_{5} y+d_{6}\right)-\lambda^{2} \cos (\lambda x)\left(\frac{n_{1}}{5040} y^{10}+\frac{n_{2}}{3024} y^{9}+\frac{n_{3}}{1680} y^{8}\right.\right. \\
& \left.\left.+\frac{n_{4}}{840} y^{7}+\frac{n_{5}}{360} y^{6}+\frac{n_{6}}{120} y^{5}+\frac{n_{7}}{24} y^{4}+\frac{n_{8}}{24} y^{4}+\frac{d_{7}}{6} y^{3}+\frac{d_{8}}{2} y^{2}+d_{9} y+d_{10}\right)\right) \\
& v_{2}=\varepsilon\left(-\lambda \sin (\lambda x)\left(l_{10} y^{4}+\frac{d_{13}}{6} y^{3}+\frac{d_{14}}{2} y^{2}+d_{15} y+d_{16}\right)-\lambda^{2} \cos (\lambda x)\left(\frac{n_{11}}{5040} y^{9}+\frac{n_{12}}{3024} y^{9}+\frac{n_{13}}{1680} y^{8}\right.\right. \\
& \left.\left.+\frac{n_{14}}{840} y^{7}+\frac{n_{15}}{360} y^{6}+\frac{n_{16}}{120} y^{5}+\frac{n_{17}}{24} y^{4}+\frac{n_{18}}{24} y^{4}+\frac{d_{17}}{6} y^{3}+\frac{d_{18}}{2} y^{2}+d_{19} y+d_{20}\right)\right) \\
& \theta_{1}=\frac{-\alpha y^{2}}{2}+c_{1} y+c_{2}+\varepsilon\left(\cos (\lambda x)\left(c_{3} y+c_{4}\right)-\lambda \sin (\lambda x)\left(P \left(m_{1} y^{7}+m_{2} y^{6}+m_{3} y^{5}+m_{4} y^{4}+m_{5} y^{3}\right.\right.\right. \\
& \left.\left.+m_{6} y^{2}\right)+c_{5} y+c_{6}\right) \\
& \theta_{2}=\frac{-\alpha y^{2}}{2}+c_{7} y+c_{8}+\varepsilon\left(\cos (\lambda x)\left(c_{9} y+c_{10}\right)-\lambda \sin (\lambda x)\left(P \left(m_{7} y^{7}+m_{8} y^{6}+m_{9} y^{5}+m_{10} y^{4}+m_{11} y^{3}\right.\right.\right. \\
& \left.\left.+m_{12} y^{2}\right)+c_{11} y+c_{12}\right) \\
& c_{1}=\frac{\alpha}{2}+m-1, \quad c_{2}=1, \quad c_{3}=\frac{\alpha}{2}+m-1, \quad c_{4}=-\frac{\alpha}{2}-m+1, \quad c_{9}=\frac{\alpha}{2}+m-1, \quad c_{10}=-\frac{\alpha}{2}-m+1, \quad c_{7}=\frac{\alpha}{2}+m-1, \quad c_{8}=1, \\
& d_{2}=0, d_{6}=0, d_{9}=0, d_{10}=0, l_{1}=\frac{G \alpha}{24}, l_{2}=\frac{-G c_{1}}{6}, l_{3}=\frac{-G c_{2}}{2}, l_{4}=\frac{G c_{3}}{24}, d_{1}=-\left(l_{1} \mathrm{y}^{*^{3}}+l_{2} \mathrm{y}^{*^{2}}+l_{3} \mathrm{y}^{*}\right), \\
& d_{5}=-\left(l_{1} \mathrm{y}^{*^{3}}+l_{2} \mathrm{y}^{*^{2}}+l_{3} \mathrm{y}^{*}\right), d_{3}=-12 l_{4} \mathrm{y}^{*}+\frac{6 d_{5}}{\mathrm{y}^{*^{2}}},
\end{aligned}
$$




$$
\begin{aligned}
& d_{4}=\frac{1}{\mathrm{y}^{*}}\left(-d_{5}-4 l_{4} \mathrm{y}^{*^{3}}-\frac{d_{3} \mathrm{y}^{*^{2}}}{2}\right), \quad l_{5}=\frac{d_{3}}{6}, l_{6}=\frac{d_{4}}{2}, l_{7}=\frac{G \alpha}{24}, l_{8}=\frac{-G c_{7}}{6}, l_{9}=\frac{-G c_{8}}{2} \text {, } \\
& d_{11}=\frac{l_{7}\left(1-\mathrm{y}^{* 4}\right)+l_{8}\left(1-\mathrm{y}^{* 3}\right)+l_{9}\left(1-\mathrm{y}^{* 2}\right)}{\mathrm{y}^{*}-1}, d_{12}=-l_{7}-l_{8}-l_{9}-d_{11}, \quad l_{10}=\frac{G c_{9}}{24}, \\
& d_{13}=\frac{-l_{10}\left(1-y^{*^{4}}-4\left(1-y^{*}\right)+2\left(1-y^{*^{3}}\right)\left(1-y^{*}\right)\right)}{\frac{\left(1-y^{*^{3}}\right)}{6}+\frac{\left(1-y^{*^{2}}\right)\left(1-y^{*}\right)}{4}-\frac{\left(1-y^{*}\right)}{2}}, d_{14}=\frac{-4 l_{10}\left(1-y^{*^{3}}\right)-\frac{d_{13}}{2}\left(1-y^{*^{2}}\right)}{\left(1-y^{*}\right)}, \quad d_{15}=-4 l_{10}-\frac{d_{13}}{2}-d_{14} \\
& , d_{16}=-l_{10}-\frac{d_{13}}{6}-\frac{d_{14}}{2}-d_{15}, \quad l_{11}=\frac{d_{13}}{6}, \quad l_{12}=\frac{d_{14}}{2}, \quad m_{1}=\frac{c_{3} l_{1}-\alpha l_{4}}{42} \\
& m_{2}=\frac{c_{3} l_{2}+c_{4} l_{1}-\alpha l_{5}+c_{1} l_{4}}{30}, m_{3}=\frac{c_{3} l_{3}+c_{4} l_{2}-\alpha l_{6}+c_{1} l_{5}}{20}, m_{4}=\frac{c_{3} d_{1}+c_{4} l_{3}-\alpha d_{5}+c_{1} l_{6}}{12}, \quad m_{5}=\frac{c_{3} d_{2}+c_{4} d_{1}-\alpha d_{6}+c_{1} d_{5}}{6}, \\
& m_{6}=\frac{c_{4} d_{2}+c_{1} d_{6}}{2}, m_{7}=\frac{c_{9} l_{7}-\alpha l_{10}}{42}, \quad m_{8}=\frac{c_{9} l_{8}+c_{10} l_{7}-\alpha l_{11}+c_{7} l_{10}}{30}, \quad m_{9}=\frac{c_{9} l_{9}+c_{10} l_{8}-\alpha l_{12}+c_{7} l_{11}}{20}, \\
& m_{10}=\frac{c_{9} d_{11}+c_{10} l_{9}-\alpha d_{15}+c_{7} d_{15}}{12}, m_{11}=\frac{c_{9} d_{12}+c_{10} d_{11}-\alpha d_{16}+c_{7} d_{15}}{6}, m_{12}=\frac{c_{10} d_{12}+c_{7} d_{16}}{2} \\
& c_{12}=i P\left(6 m_{7} \mathrm{y}^{*^{7}}+5 m_{8} \mathrm{y}^{*^{6}}+4 m_{9} \mathrm{y}^{*^{5}}+3 m_{10} \mathrm{y}^{*^{4}}+2 m_{11} \mathrm{y}^{*^{3}}+m_{12} \mathrm{y}^{*^{2}}\right)-6 m_{1} \mathrm{y}^{*^{7}}-5 m_{2} \mathrm{y}^{*^{6}}-4 m_{3} \mathrm{y}^{* 5} \\
& -3 m_{4} \mathrm{y}^{* 4}-2 m_{5} \mathrm{y}^{* 3}-m_{6} \mathrm{y}^{* 2} \\
& c_{11}=-P i\left(m_{7}+m_{8}+m_{9}+m_{10}+m_{11}+m_{12}\right)-c_{12} \\
& c_{5}=i P\left(7 m_{7} \mathrm{y}^{*^{6}}+6 m_{8} \mathrm{y}^{*^{5}}+5 m_{9} \mathrm{y}^{*^{4}}+4 m_{10} \mathrm{y}^{*^{3}}+3 m_{11} \mathrm{y}^{*^{2}}+2 m_{12} \mathrm{y}^{*}\right)-i P\left(7 m_{1} \mathrm{y}^{*^{6}}+6 m_{2} \mathrm{y}^{*^{5}}+5 m_{3} \mathrm{y}^{*^{4}}\right. \\
& \left.+4 m_{4} \mathrm{y}^{*^{3}}+3 m_{5} \mathrm{y}^{*^{2}}+2 m_{6} \mathrm{y}^{*}\right)+c_{11} \\
& n_{1}=7 G P m_{1}, \quad n_{2}=6 l_{2} l_{4}-6 l_{1} l_{5}+6 G P m_{2}, \quad n_{3}=10 l_{3} l_{4}-10 l_{1} l_{6}+5 G P m_{3} \\
& n_{4}=12 d_{1} l_{4}+4 l_{5} l_{3}-4 l_{2} l_{6}-12 l_{1} d_{5}+24 l_{4} l_{2} K-24 l_{1} l_{5} K+4 G P m_{4} \\
& n_{5}=12 l_{4} d_{2}+6 l_{5} d_{1}-6 l_{2} d_{5}-12 l_{1} d_{6}+24 l_{3} l_{4} K-24 l_{1} l_{6} K+3 G P m_{5} \\
& n_{6}=6 l_{5} d_{2}+2 l_{6} d_{1}-6 l_{2} d_{6}-2 l_{3} d_{5}+24 d_{1} l_{4} K-24 l_{1} d_{5} K+2 G P m_{6}, n_{7}=2 l_{6} d_{2}-2 l_{3} d_{6}+24 K l_{4} d_{2}-24 K l_{1} d_{6}, n_{8}=G c_{5} \\
& d_{7}=-\frac{n_{1} \mathrm{y}^{* 9}}{105}-\frac{n_{2} \mathrm{y}^{* 8}}{72}-\frac{3 n_{3} \mathrm{y}^{* 7}}{140}-\frac{n_{4} \mathrm{y}^{* 6}}{28}-\frac{n_{5} \mathrm{y}^{* 5}}{15}-\frac{3 n_{6} \mathrm{y}^{* 4}}{20}-\frac{n_{7} \mathrm{y}^{* 3}}{2}-\frac{n_{8} \mathrm{y}^{*}}{2} \\
& d_{8}=-\frac{n_{1} \mathrm{y}^{* 8}}{2520}-\frac{n_{2} \mathrm{y}^{* 7}}{1512}-\frac{n_{3} \mathrm{y}^{* 6}}{840}-\frac{n_{4} \mathrm{y}^{* 5}}{420}-\frac{n_{5} \mathrm{y}^{* 4}}{180}-\frac{n_{6} \mathrm{y}^{* 3}}{60}-\frac{n_{7} \mathrm{y}^{* 2}}{12}-\frac{n_{8} \mathrm{y}^{* 2}}{12}-\frac{d_{7} \mathrm{y}^{*}}{3} \\
& n_{11}=7 G P m_{7}, n_{12}=6 l_{8} l_{10}-6 l_{7} l_{11}+6 G P m_{8}, n_{13}=10 l_{9} l_{10}-10 l_{7} l_{12}+5 G P m_{9} \\
& n_{14}=12 d_{11} l_{10}+4 l_{9} l_{11}-4 l_{8} l_{12}-12 l_{7} d_{15}+24 l_{8} l_{10} K-24 l_{7} l_{11} K+4 G P m_{10} \\
& n_{15}=12 l_{10} d_{12}+6 l_{11} d_{11}-6 l_{8} d_{15}-12 l_{7} d_{16}+24 l_{9} l_{10} K-24 l_{7} l_{12} K+3 G P m_{11} \\
& n_{16}=6 l_{11} d_{12}+2 l_{12} d_{11}-6 l_{8} d_{16}-2 l_{9} d_{15}+24 d_{11} l_{10} K-24 l_{7} d_{15} K+2 G P m_{12}, n_{17}=2 l_{12} d_{12}-2 l_{9} d_{16}+24 K l_{10} d_{12}-24 K l_{7} d_{16} \text {, } \\
& n_{18}=G c_{11}, d_{14}=\left(-4 l_{10}\left(1-y^{* 4}\right)-\frac{d_{13}}{2}\left(1-y^{* 3}-1\right)\right) /\left(1-y^{*}\right), d_{15}=-4 l_{10}-\frac{d_{13}}{2}-d_{14}, d_{16}=-l_{10}-\frac{d_{13}}{6}-\frac{d_{14}}{2}-d_{15} \text {, } \\
& Z_{1}=\frac{1}{504} n_{11}\left(1-y^{*^{9}}\right)+\frac{1}{336} n_{12}\left(1-y^{*^{*^{8}}}\right)+\frac{1}{210} n_{13}\left(1-y^{*^{7}}\right)+\frac{1}{120} n_{14}\left(1-y^{*^{6}}\right)+\frac{1}{60} n_{15}\left(1-y^{*^{5}}\right) \\
& +\frac{1}{24} n_{16}\left(1-y^{*^{4}}\right)+\frac{1}{6} n_{17}\left(1-y^{*^{3}}\right)+\frac{1}{6} n_{18}\left(1-y^{*^{2}}\right)
\end{aligned}
$$




$$
\begin{aligned}
& Z_{2}=n_{11}\left(\frac{1}{5040}\left(1-y^{*^{10}}\right)-\frac{1}{504}\left(1-y^{*}\right)\right)+n_{12}\left(\frac{1}{3024}\left(1-y^{*^{9}}\right)-\frac{1}{336}\left(1-y^{*}\right)\right) \\
& +n_{13}\left(\frac{1}{1680}\left(1-y^{*^{8}}\right)-\frac{1}{210}\left(1-y^{*}\right)\right)+n_{14}\left(\frac{1}{840}\left(1-y^{*^{7}}\right)-\frac{1}{120}\left(1-y^{*}\right)\right)+n_{15}\left(\frac{1}{360}\left(1-y^{*^{6}}\right)-\frac{1}{60}\left(1-y^{*}\right)\right) \\
& +n_{16}\left(\frac{1}{120}\left(1-y^{*^{5}}\right)-\frac{1}{24}\left(1-y^{*}\right)\right)+n_{17}\left(\frac{1}{24}\left(1-y^{*^{4}}\right)-\frac{1}{6}\left(1-y^{*}\right)\right)+n_{18}\left(\frac{1}{24}\left(1-y^{*^{3}}\right)-\frac{1}{6}\left(1-y^{*}\right)\right) \\
& d_{17}=-Z_{2}-Z_{1} \frac{1}{2}\left(1-y^{*}\right) /\left(\frac{1}{6}\left(1-y^{*^{3}}\right)+\frac{1}{4}\left(1-y^{*^{2}}\right)\left(1-y^{*}\right)-\frac{1}{2}\left(1-y^{*}\right)\right), \quad d_{18}=-Z_{1}-\frac{d_{17}}{2}\left(1-y^{*^{2}}\right) /\left(1-y^{*}\right) \\
& d_{19}=-\left(n_{11} \frac{y^{*^{9}}}{504}+n_{12} \frac{y^{*^{8}}}{336}+n_{13} \frac{y^{*^{7}}}{210}+n_{14} \frac{y^{*^{6}}}{120}+n_{15} \frac{y^{*^{5}}}{60}+n_{16} \frac{y^{*^{4}}}{24}+n_{17} \frac{y^{*^{3}}}{6}+n_{18} \frac{y^{*^{3}}}{6}+d_{17} \frac{y^{*^{2}}}{2}+d_{18} y^{*}\right) \\
& d_{20}=-\left(\frac{n_{11}}{5040}+\frac{n_{12}}{3024}+\frac{n_{13}}{1680}+\frac{n_{14}}{840}+\frac{n_{15}}{360}+\frac{n_{16}}{120}+\frac{n_{17}}{24}+\frac{n_{18}}{24}+\frac{d_{17}}{6}+\frac{d_{18}}{2}+d_{19}\right)
\end{aligned}
$$

Case 2a: Comparison of the Solutions with Salah El Din (2002) in the presence of baffle

$$
\begin{aligned}
& \theta_{10}=y-\frac{1}{2}, \theta_{20}=y-\frac{1}{2} \\
& u_{10}=-\frac{G}{2}\left(\frac{y^{3}}{3}-\frac{y^{2}}{2}\right)+d_{1} y+d_{2}, u_{20}=-\frac{G}{2}\left(\frac{y^{3}}{3}-\frac{y^{2}}{2}\right)+d_{3} y+d_{4} \\
& d_{1}=-\frac{G}{2}\left(\frac{y^{*^{2}}}{3}-\frac{y^{*}}{2}\right), d_{2}=0, d_{3}=-c_{8}-\frac{G}{12}, d_{4}=\frac{G}{2\left(1-y^{*}\right)}\left(\frac{y^{*^{3}}}{3}-\frac{y^{*^{2}}}{2}+\frac{y^{*}}{6}\right)
\end{aligned}
$$

\section{Case 2b: Comparison of the Solutions with Rita and Alok (2000) in the absence of baffle}

1. Shifting the baffle to the left wall and comparing the solutions of stream-1 with Rita and Alok (2000).

$$
\begin{aligned}
& \theta_{10}=\frac{-\alpha y^{2}}{2}+c_{1} y+c_{2} \\
& c_{2}=1, c_{1}=m+\frac{\alpha}{2}-1 \\
& u_{10}=l_{1} y^{4}+l_{2} y^{3}+l_{3} y^{2}+d_{1} y+d_{2} \\
& l_{1}=\frac{G \alpha}{24}, l_{2}=\frac{-G c_{1}}{6}, l_{3}=\frac{-G c_{2}}{2}, d_{1}=-l_{1}-l_{2}-l_{3}, d_{2}=0 \\
& t_{10}=c_{3} y+c_{4} \\
& c_{4}=-m-\frac{\alpha}{2}+1, c_{3}=m+\frac{\alpha}{2}-1 \\
& \psi_{10}=l_{4} y^{4}+\frac{d_{3}}{6} y^{3}+\frac{d_{4}}{2} y^{2}+d_{5} y+d_{6} \\
& l_{4}=\frac{G c_{3}}{24}, d_{6}=0, d_{5}=-l_{1}-l_{2}-l_{3}, d_{3}=-12 l_{4}+6 d_{5}, d_{4}=-d_{1}-\frac{d_{3}}{2}-4 l_{4} \\
& t_{11}=P i\left(m_{1} y^{7}+m_{2} y^{6}+m_{3} y^{5}+m_{4} y^{4}+m_{5} y^{3}+m_{6} y^{2}\right)+c_{5} y+c_{6} \\
& m_{1}=\frac{c_{3} l_{1}-\alpha l_{4}}{42}, m_{2}=\frac{c_{3} l_{2}+c_{4} l_{1}-\alpha l_{5}+c_{1} l_{4}}{30}, m_{3}=\frac{c_{3} l_{3}+c_{4} l_{2}-\alpha l_{6}+c_{1} l_{5}}{20}, m_{4}=\frac{c_{3} d_{1}+c_{4} l_{3}-\alpha d_{5}+c_{1} l_{6}}{12}, \\
& m_{5}=\frac{c_{3} d_{2}+c_{4} d_{1}-\alpha d_{6}+c_{1} d_{5}}{c_{6}=}, m_{6}=\frac{c_{4} d_{2}+c_{1} d_{6}}{2} \\
& c_{5}=-P i\left(m_{1}+m_{2}+m_{3}+m_{4}+m_{5}+m_{6}\right)
\end{aligned}
$$




$$
\begin{aligned}
& \psi_{11}=i\left(\frac{n_{1}}{5040} y^{10}+\frac{n_{2}}{3024} y^{9}+\frac{n_{3}}{1680} y^{8}+\frac{n_{4}}{840} y^{7}+\frac{n_{5}}{360} y^{6}+\frac{n_{6}}{120} y^{5}+\frac{n_{7}}{24} y^{4}\right)+\frac{n_{8}}{24} y^{4}+\frac{d_{7}}{6} y^{3}+\frac{d_{8}}{2} y^{2} \\
& +d_{9} y+d_{10} \\
& n_{1}=7 G P m_{1}, n_{2}=6 l_{2} l_{4}-6 l_{1} l_{5}+6 G P m_{2}, n_{3}=10 l_{3} l_{4}-10 l_{1} l_{6}+5 G P m_{3}, \\
& n_{4}=12 d_{1} l_{4}+4 l_{5} l_{3}-4 l_{2} l_{6}-12 l_{1} d_{5}+24 l_{4} l_{2} K-24 l_{1} l_{5} K+4 G P m_{4}, n_{5}=12 l_{4} d_{2}+6 l_{5} d_{1}-6 l_{2} d_{5}-12 l_{1} d_{6}+24 l_{3} l_{4} K-24 l_{1} l_{6} K+3 G P m_{5} \\
& n_{6}=6 l_{5} d_{2}+2 l_{6} d_{1}-6 l_{2} d_{6}-2 l_{3} d_{5}+24 d_{1} l_{4} K-24 l_{1} d_{5} K+2 G P m_{6}, n_{7}=2 l_{6} d_{2}-2 l_{3} d_{6}+24 K l_{4} d_{2}-24 K l_{1} d_{6}, n_{8}=G c_{5} \\
& d_{7}=-i\left(\frac{n_{1}}{105}+\frac{n_{2}}{72}+\frac{3 n_{3}}{140}+\frac{n_{4}}{28}+\frac{n_{5}}{15}+\frac{3 n_{6}}{20}+\frac{n_{7}}{2}\right)-\frac{n_{8}}{2}, \\
& d_{8}=-i\left(\frac{n_{1}}{2520}+\frac{n_{2}}{1512}+\frac{n_{3}}{840}+\frac{n_{4}}{420}+\frac{n_{5}}{180}+\frac{n_{6}}{60}+\frac{n_{7}}{12}\right)-\frac{n_{8}}{12}-\frac{d_{7}}{3}, d_{9}=0, d_{10}=0
\end{aligned}
$$

2. Shifting the baffle to the right wall and comparing the solutions of stream-1 with Rita and Alok (2000).

$$
\begin{aligned}
& \theta_{20}=\frac{-\alpha y^{2}}{2}+c_{7} y+c_{8} \\
& c_{8}=1, c_{7}=m+\frac{\alpha}{2}-1 \\
& u_{20}=l_{7} y^{4}+l_{8} y^{3}+l_{9} y^{2}+d_{11} y+d_{12} \\
& l_{7}=\frac{G \alpha}{24}, l_{8}=\frac{-G c_{1}}{6}, l_{9}=\frac{-G c_{2}}{2}, d_{11}=-l_{7}-l_{8}-l_{9}, d_{12}=0 \\
& t_{20}=c_{9} y+c_{10} \\
& c_{10}=-m-\frac{\alpha}{2}+1, c_{9}=m+\frac{\alpha}{2}-1 \\
& \psi_{20}=l_{10} y^{4}+\frac{d_{13}}{6} y^{3}+\frac{d_{14}}{2} y^{2}+d_{15} y+d_{16} \\
& l_{10}=\frac{G c_{3}}{24}, d_{16}=0, d_{15}=d_{11}, d l_{3}=-12 l_{10}+6 d_{15}, d_{14}=-d_{15}-\frac{d_{13}}{2}-4 l_{10} \\
& t_{21}=P i\left(m_{7} y^{7}+m_{8} y^{6}+m_{9} y^{5}+m_{10} y^{4}+m_{11} y^{3}+m_{12} y^{2}\right)+c_{11} y+c_{12} \\
& m_{7}=\frac{c_{9} l_{7}-\alpha l_{10}}{42}, \quad m_{8}=\frac{c_{9} l_{8}+c_{10} l_{7}-\alpha l_{11}+c_{7} l_{10}}{30}, \quad m_{9}=\frac{c_{9} l_{9}+c_{10} l_{8}-\alpha l_{12}+c_{7} l_{11}}{20}, m_{10}=\frac{c_{9} d_{11}+c_{10} l_{9}-\alpha d_{15}+c_{7} d_{15}}{12} \text {, } \\
& m_{11}=\frac{c_{9} d_{12}+c_{10} d_{11}-\alpha d_{16}+c_{7} d_{15}}{6}, m_{12}=\frac{c_{10} d_{12}+c_{7} d_{16}}{2} \\
& c_{12}=0, c_{11}=-P i\left(m_{7}+m_{8}+m_{9}+m_{10}+m_{11}+m_{12}\right) \\
& \psi_{21}=i\left(\frac{n_{11}}{5040} y^{10}+\frac{n_{12}}{3024} y^{9}+\frac{n_{13}}{1680} y^{8}+\frac{n_{14}}{840} y^{7}+\frac{n_{15}}{360} y^{6}+\frac{n_{16}}{120} y^{5}+\frac{n_{17}}{24} y^{4}\right)+\frac{n_{18}}{24} y^{4}+\frac{d_{17}}{6} y^{3}+\frac{d_{18}}{2} y^{2} n_{11}=7 G P m_{7} \text {, } \\
& +d_{19} y+d_{20} \\
& n_{12}=6 l_{8} l_{10}-6 l_{7} l_{11}+6 G P m_{8}, n_{13}=10 l_{9} l_{10}-10 l_{7} l_{12}+5 G P m_{9} \text {, } \\
& n_{14}=12 d_{11} l_{10}+4 l_{9} l_{11}-4 l_{8} l_{12}-12 l_{7} d_{15}+24 l_{8} l_{10} K-24 l_{7} l_{11} K+4 G P m_{10} \text {, } \\
& n_{15}=12 l_{10} d_{12}+6 l_{11} d_{11}-6 l_{8} d_{15}-12 l_{7} d_{16}+24 l_{9} l_{10} K-24 l_{7} l_{12} K+3 G P m_{11} \text {, } \\
& n_{16}=6 l_{11} d_{12}+2 l_{12} d_{11}-6 l_{8} d_{16}-2 l_{9} d_{15}+24 d_{11} l_{10} K-24 l_{7} d_{15} K+2 G P m_{12}, n_{17}=2 l_{12} d_{12}-2 l_{9} d_{16}+24 K l_{10} d_{12}-24 K l_{7} d_{16} \text {, } \\
& n_{18}=G c_{11} \quad d_{17}=-i\left(\frac{n_{11}}{105}+\frac{n_{12}}{72}+\frac{3 n_{13}}{140}+\frac{n_{14}}{28}+\frac{n_{15}}{15}+\frac{3 n_{16}}{20}+\frac{n_{17}}{2}\right)-\frac{n_{18}}{2} \text {, } \\
& d_{18}=-i\left(\frac{n_{11}}{2520}+\frac{n_{12}}{1512}+\frac{n_{13}}{840}+\frac{n_{14}}{420}+\frac{n_{15}}{180}+\frac{n_{16}}{60}+\frac{n_{17}}{12}\right)-\frac{n_{18}}{12}-\frac{d_{17}}{3}, d_{19}=0, d_{20}=0
\end{aligned}
$$




\section{Acknowledgments}

One of the authors J.C. Umavathi would like to thank UGC-New Delhi for the financial support under UGC-Major Research Project.

\section{References}

Aung, W. and Worku, G., 1986. Theory of fully developed, combined convection including flow reversal, J. Heat Transfer, Vol. 108, pp. 485-488.

Blancher, S., Creff, R. and Quere, P.L., 1998. Effect of tollmien schlichting wave on convective heat transfer in a wavy channel part I: Linear analysis, Int. J. Heat Fluid Flow, Vol. 19, pp. 39-48.

Bőhme, G., 1981. Non-Newtonian fluid mechanics, North Holland Amsterdam.

Burns, J.C. and Parks, T., 1967. Peristaltic motion, J. Fluid Mech., Vol.29, pp. 405-416.

Chang, T.S., and Shiau, Y.H., 2005. Flow pulsation and baffle's effects on the opposing mixed convection in a vertical channel, Int. J. Heat Mass Transfer, Vol. 48, pp. 4190-4204.

Chen, Z.D. and Chen, J.J.J., 1998. Local heat transfer for oscillatory flow in the presence of a single baffle within a channel, Chem. Eng. Sci., Vol. 53, pp. 3177-3180.

Cheng, C.H., Kuo, H.S and Huang, W.H., 1989. Laminar fully developed forced-convection flow within an asymmetric heated horizontal double-passage channel, Applied. Energy, Vol. 33, pp. 265-286.

Chiu, C.P. and Chou, H.M. 1994. Transient analysis of natural convection along a vertical wavy surface in micropolar fluids, Int. J. Energy Science, Vol. 32, pp 19-33.

Dutta, P and Hossain, A., 2005. Internal cooling augmentation in rectangular channel using two inclined baffles, Int. J. Heat Fluid Flow, Vol. 26, pp. 223-232.

Jang, J.H., Yan, W.M. and Liu, H.C., 2003. Natural convection heat and mass transfer along a vertical wavy surface, Int. J. Heat Mass Transfer, Vol. 46, pp. 1075-1083.

Greiner, M., Chen, R.F. and Wirtz, R.A., 1991. Enhanced heat transfer/pressure drop measured from a flat surface in a grooved channel, ASME J. Heat Transfer, Vol. 113, pp. 498-501.

Guo, Z.Y., Li, D.Y. and Wang, B.X., 1998. A novel concept for convective heat transfer enhancement, Int. J. Heat Mass Transfer, Vol. 41, pp. 2221-2225.

Huilgol, R.R. and Phan-Thien, N., 1997. Fluid mechanics of viscoelasticity, Elsevier, Amsterdam.

Malashetty, M.S., Umavathi, J.C. and Leela, V., 2001. Magnetoconvective flow and heat transfer between vertical wavy wall and a parallel flat wall, Int. J. of Applied Mechanics and Engineering, Vol. 6, No. 2, pp. 437-456.

Ostrich, S., 1952. An analysis of laminar free-convection flow and heat transfer about a flat plate parallel to the direction of the generating body force, NACA, TN -2635, Accession number -93R12788, Documentation ID-19930083498.

Rees, D.A.S. and Pop, I., 1994. A note on free convection along a vertical wavy surface in a porous medium, ASME J. Heat Transfer, Vol. 116, pp. 505-508.

Rees, D.A.S. and Pop, I., 1995. Free convection induced by a vertical wavy surface with uniform heat flux in porous medium, ASME J. Heat Transfer, Vol. 117, pp. 547-550.

Rita Choudhury and Alok Das, 2000. Free convection flow of a non-Newtonian fluid in a vertical channel, Defence Science Journal, Vol. 50, pp. 37-44.

Salah El-Din, M.M., 1994. Fully developed laminar convection in a vertical double-passage channel, Appl. Energy, Vol. 47, pp. 69-75.

Salah El-Din, M.M., 2002. Effect of viscous dissipation on fully developed laminar mixed convection in a vertical double-passage channel, Int. J. Therm. Sci, Vol. 41, pp. 253-259.

Selvarajan, S., Tulapurkara, E.G. and Ram, V.V., 1998. A numerical study of flow through wavy-walled channels, J. Numer. Meth. Fluids, Vol. 26, pp. 519-531.

Umavathi, J.C., Mallikarjun B Patil and Pop, I., 2006. On laminar mixed convection flow in a vertical porous stratum with symmetric wall heating conditions, Int. J. Transp. Phenom., Vol. 8, pp. 127-140.

Vajravelu, K., 1980. Fluid flow and heat transfer in horizontal wavy channels. Acta Mechanica, Vol. 35, pp. 245-258.

Walters, K., 1960. The motion of an elastico-viscous liquid contained between coaxial cylinders (II), Quart. J. Mech. Appl. Maths, Vol. 13, pp. 444-461.

Walters, K., 1962. The solution of flow problems in the case of materials with memories, Journal Mecanique, Vol. 1, pp. 474-478.

Wang, G and Vanka, S.P., 1995. Convective heat transfer in periodic wavy passages, Int. J. Heat Mass Transfer, Vol. 38, pp. 3219-3230.

Yao, L.S., 1983. Free and forced convection between in the entry region of a heated vertical channel, Int. J. Heat Mass Transfer, Vol. 26, pp. 65-72. 


\section{Biographical notes}

J. Prathap Kumar received M. Phil. degree from Mangalore University Mangalore in 1990 and Ph. D degree from Gulbarga University Gulbarga India in 2003. $\mathrm{He}$ is a associate professor in the department of Mathematics Gulbarga University, Gulbarga, Karnataka, India. His research interest includes heat and mass transfer, dispersion, baffles for flow through various geometries of one and two fluids models. He has published more than 20 papers in referred International journals. He has also presented 5 research articles in national conferences.

J. C. Umavathi received $\mathrm{Ph}$. D degree from Gulbarga University Gulbarga India in 1992. She is a Professor in the department of Mathematics, Gulbarga University, Gulbarga, Karnataka, India. Her research interest includes heat and mass transfer of multiple (Newtonian and non-Newtonian) fluids through channels and rectangular ducts, numerical simulation using Finite differences and Range-Kutta Gill method, magnetohydradynamics, flow through porous media she has published more than 60 papers in referred International journals. She has also presented more than 20 research articles in National and International conferences. She currently dealing with few projects sponsored by Government of India.

P.M. Hiremath received Post-Graduation in Mathematics from Gulbarga University, Gulbarga, Karnataka, India in 1983. She is working as Assistant Professor in Mathematics at B.L.D.E.A.'s College of Engineering and Technology Bijapur, Karnataka. Her research interest includes heat and mass transfer through channels.

Received January 2011

Accepted April 2011

Final acceptance in revised form May 2011 